\title{
3D kinematics and age distribution of the open cluster population ${ }^{\star}$
}

\author{
Y. Tarricq ${ }^{1}$, C. Soubiran ${ }^{1}$, L. Casamiquela ${ }^{1}$, T. Cantat-Gaudin ${ }^{2}$, L. Chemin ${ }^{3}$, F. Anders ${ }^{2}$, T. Antoja ${ }^{2}$, \\ M. Romero-Gómez ${ }^{2}$, F. Figueras ${ }^{2}$, C. Jordi² ${ }^{2}$ A. Bragaglia ${ }^{4}$, L. Balaguer-Núñez ${ }^{2}$, R. Carrera ${ }^{5}$, A. Castro-Ginard ${ }^{2}$, \\ A. Moitinho ${ }^{6}$, P. Ramos ${ }^{2}$, and D. Bossini ${ }^{7}$
}

\author{
${ }^{1}$ Laboratoire d'Astrophysique de Bordeaux, Univ. Bordeaux, CNRS, B18N, allée Geoffroy Saint-Hilaire, 33615 Pessac, France \\ e-mail: yoann.tarricq@u-bordeaux.fr \\ 2 Institut de Ciéncies del Cosmos, Universitat de Barcelona (IEEC-UB), Martí i Franqués 1, 08028 Barcelona, Spain \\ 3 Centro de Astronomía, Universidad de Antofagasta, Avda. U.de Antofagasta 02800, Antofagasta, Chile \\ 4 INAF-Osservatorio di Astrofisica e Scienza dello Spazio, via P. Gobetti 93/3, 40129 Bologna, Italy \\ 5 INAF-Osservatorio Astronomico di Padova, vicolo dell'Osservatorio 5, 35122 Padova, Italy \\ ${ }^{6}$ CENTRA, Faculdade de Ciencias, Universidade de Lisboa, Ed. C8, Campo Grande, 1749-016 Lisboa, Portugal \\ 7 CAUP - Centro de Astrofisica da Universidade do Porto, Rua das Estrelas, Porto, Portugal
}

Received 10 September 2020 / Accepted 1 December 2020

\begin{abstract}
Context. Open clusters (OCs) trace the evolution of the Galactic disc with great accuracy. Gaia and large ground-based spectroscopic surveys make it possible to determine their properties and study their kinematics with unprecedented precision.

Aims. We study the kinematical behaviour of the OC population over time. We take advantage of the latest age determinations of OCs to investigate the correlations of the $6 \mathrm{D}$ phase-space coordinates and orbital properties with age. The phase-space distribution, age-velocity relation, and action distribution are compared to those of field stars. We also investigate the rotation curve of the Milky Way traced by OCs, and we compare it to that of other observational or theoretical studies.

Methods. We gathered nearly 30000 radial velocity (RV) measurements of OC members from both Gaia-RVS data and ground-based surveys and catalogues. We computed the weighted mean RV, Galactic velocities, and orbital parameters of 1382 OCs. We investigated their distributions as a function of age and by comparison to field stars.

Results. We provide the largest RV catalogue available for OCs, half of it based on at least three members. Compared to field stars, we note that OCs are not on exactly the same arches in the radial-azimuthal velocity plane, while they seem to follow the same diagonal ridges in the Galactic radial distribution of azimuthal velocities. Velocity ellipsoids in different age bins all show a clear anisotropy. The heating rate of the OC population is similar to that of field stars for the radial and azimuthal components, but it is significantly lower for the vertical component. The rotation curve drawn by our sample of clusters shows several dips that match the wiggles derived from nonaxisymmetric models of the Galaxy. From the computation of orbits, we obtain a clear dependence of the maximum height and eccentricity on age. Finally, the orbital characteristics of the sample of clusters as shown by the action variables follow the distribution of field stars. The additional age information of the clusters indicates some (weak) age dependence of the known moving groups.
\end{abstract}

Key words. open clusters and associations: general - Galaxy: kinematics and dynamics - Galaxy: disk stars: kinematics and dynamics

\section{Introduction}

The study of kinematical properties of the Milky Way open clusters (OCs) has a long tradition. Their motion can be used to understand the Milky Way gravitational potential and the various perturbations that act on the structure and dynamics of the Galactic disc. The solar neighbourhood is known to be clumpy (e.g., Eggen 1996; Dehnen \& Binney 1998; Antoja et al. 2012), and the relation of observed substructures to some OCs, in particular the Hyades, is established (Eggen 1958; Chereul et al. 1999). While stellar streams were initially thought to be remnants of star clusters, their origin is now thought to be the result of the disruption of star clusters or of dynamical origin, such as the resonant trapping by the bar and spiral arms (Famaey et al. 2008) or the passage of the Sagittarius dwarf galaxy within the

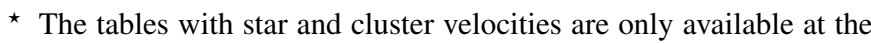
CDS via anonymous ftp to cdsarc.u-strasbg. fr (130.79.128.5) or via http://cdsarc.u-strasbg.fr/viz-bin/cat/J/A+A/647/ A19
}

Galactic plane (Monari et al. 2018; Khanna et al. 2019). Stellar streams could also originate from a combination of these processes. Gaia DR2 data (Gaia Collaboration 2018b) revealed the complexity of the local velocity distribution with unprecedented resolution, showing in particular that the velocity distribution of nearby OCs overlaps prominent arched overdensities of moving groups well (Gaia Collaboration 2018d).

Gaia DR2 has considerably furthered the study of OCs with the determination of new memberships for an unprecedented number of stars and clusters. A first large catalogue was published by Cantat-Gaudin et al. (2018), who systematically searched for members around the $\sim 3300$ catalogue OCs (mostly from Dias et al. 2002 and Kharchenko et al. 2013 studies), only based on Gaia DR2 positions, parallaxes, and proper motions. Membership probabilities were computed for 400 000 stars, and among other parameters, the most probable distances were determined for 1229 OCs. A large fraction of candidate OCs could not be confirmed by Gaia or was proved to correspond to chance alignment. The number of 
OC candidates has also significantly increased, either through serendipitous discoveries in Gaia DR2 (Cantat-Gaudin et al. 2018; Ferreira et al. 2019), or as the result of systematic searches (Castro-Ginard et al. 2018, 2019, 2020; Cantat-Gaudin et al. 2019; Sim et al. 2019; Liu \& Pang 2019). An updated catalogue of membership probabilities for 1481 OCs has been provided by Cantat-Gaudin \& Anders (2020). The physical and kinematical properties of OCs has also been revisited based on Gaia. Using the memberships from Cantat-Gaudin et al. (2018) and only Gaia data, Bossini et al. (2019) determined the age, distance modulus, and extinction of 269 OCs, and Soubiran et al. (2018a) computed the 6D phase-space information of 861 clusters. Carrera et al. (2019) increased the number of OCs with full 6D phase-space information by 145 by searching for members in the GALAH (GALactic Archaeology with HERMES) and APOGEE (Apache Point Observatory Galactic Evolution Experiment) spectroscopic surveys. The vertical distribution of young clusters was found to be very flat, with a dispersion of vertical velocities of $5 \mathrm{~km} \mathrm{~s}^{-1}$, while clusters older than $1 \mathrm{Gyr}$ span distances to the Galactic plane of up to $1 \mathrm{kpc}$ with a vertical velocity dispersion of $14 \mathrm{~km} \mathrm{~s}^{-1}$, typical of the thin disc. Recently, Cantat-Gaudin et al. (2020) derived physical properties of all known OCs identified in the Gaia data in a homogeneous fashion, with a method based on isochrone fitting and an artificial neural network. They finally assembled 1867 clusters with reliable ages.

The paper is organised as follows. Section 2 describes the collection of individual RVs gathered from Gaia DR2 (Sartoretti et al. 2018; Katz et al. 2019) and from ground-based resources that we used to build two catalogues of mean RVs for each star and each cluster. In Sect. 3, 6D galactocentric coordinates are computed. We compare the resulting distributions with those of nearby field stars. We evaluate the variation in different coordinates with cluster age, and we determine the age velocity relation of the population. We investigate how OCs follow the theoretical rotation curve of the Galactic disc. Orbital parameters and actions are described in Sect. 4 and are used to revisit the link between OCs of different ages and the phase-space substructures of the disc. The conclusions of this study are summarized in Sect. 5.

\section{Radial velocities}

\subsection{Input data}

We took advantage of the catalogue of OCs by Cantat-Gaudin et al. (2020), which provides a list of probable members for 2017 OCs that the authors used to estimate ages. The OC memberships mostly come from Cantat-Gaudin \& Anders (2020), who used the unsupervised classification scheme UPMASK (KroneMartins \& Moitinho 2014; Cantat-Gaudin et al. 2018). These authors also applied UPMASK to the recently discovered University of Barcelona clusters (UBC, Castro-Ginard et al. 2018, 2019, 2020) and to the clusters discovered by Liu \& Pang (2019). For the Hyades and Coma Berenices, they adopted the list of members published by Gaia Collaboration (2018a) because UPMASK cannot recover members for populated clusters that are too extended on the sky.

Based on this list of members that contains $\sim 475000$ stars, we gathered all the RV measurements available for them in various surveys and catalogues. Our main source was Gaia DR2, which includes RVs for about seven million stars (Sartoretti et al. 2018; Katz et al. 2019). We also queried several catalogues from large spectroscopic surveys in addition to Gaia: the latest public ver-
Table 1. Number of stars in common in the catalogue of Cantat-Gaudin et al. (2020) and catalogues of RVs, with the corresponding median uncertainty (in $\mathrm{km} \mathrm{s}^{-1}$ ), after the quality cuts described in the text.

\begin{tabular}{lcc}
\hline \hline Survey/catalogue & $N_{\text {stars }}$ & Median RV error \\
\hline RVS & 10741 & 1.33 \\
GES & 9894 & 0.50 \\
APO & 3212 & 0.21 \\
GAL & 1724 & 0.59 \\
MER & 1313 & 0.28 \\
RAV & 386 & 1.59 \\
OCC & 187 & 0.04 \\
S18 & 138 & 0.002 \\
NOR & 73 & 0.60 \\
WOR & 10 & 0.20 \\
\hline
\end{tabular}

Notes. Catalogues are designated by short names as defined below the table. RVS: Gaia DR2; GES: Gaia ESO survey; APO: APOGEE DR16; GAL: GALAH DR3; MER: Mermilliod et al. (2008, 2009); RAV: RAVE DR6; OCC: OCCASO Casamiquela et al. (2016); S18: Soubiran et al. (2018b); NOR: Nordström et al. (2004); WOR: Worley et al. (2012).

sion of the Gaia-ESO survey (Randich et al. 2013), APOGEE DR16 (Ahumada et al. 2020), RAVE (Radial Velocity Experiment) DR6 (Steinmetz et al. 2020), and GALAH DR3 (Buder et al. 2020). We did not consider LAMOST (Large Sky Area MultiObject Fibre Spectroscopic Telescope; Cui et al. 2012; Xiang et al. 2015) because its RV precision and accuracy $\left(\sim 5 \mathrm{~km} \mathrm{~s}^{-1}\right)$ are not at the same level. We included RVs derived in by the OCCASO (Open Cluster Chemical Abundances from Spanish Observatories) survey (Casamiquela et al. 2016, Carrera et al., in prep.). We also considered the RV catalogues by Soubiran et al. (2018b), Mermilliod et al. (2009, 2008), Worley et al. (2012), and Nordström et al. (2004). Several quality cuts were applied to individual measurements. For RAVE we applied the criteria suggested by Steinmetz et al. (2020) by selecting stars that met $\mid$ correctionRV $\mid<10 \mathrm{~km} \mathrm{~s}^{-1}, \sigma_{\mathrm{RV}}<8 \mathrm{~km} \mathrm{~s}^{-1}$ and correlationCoeff $>10$. For APOGEE, we rejected the stars flagged as VERY_BRIGHT_NEIGHBOR, BAD_PIXELS or LOW_SNR as recommended in the online documentation of the survey. For Gaia RVS we filtered the erroneous RVs found by Boubert et al. (2019). Despite these cuts, individual measurements still had large uncertainties that were incompatible with the precision we required. This convinced us to filter out all the individual RVs with uncertainties larger than $8 \mathrm{~km} \mathrm{~s}^{-1}$, which is the same cut as for RAVE. The rejected values represent $6 \%$ of the full set, and $10 \%$ for the RVS set. Then we realised that 43 stars had $|\mathrm{RV}|>$ $200 \mathrm{~km} \mathrm{~s}^{-1}$, which were mainly OB-type, Wolf-Rayet, or spectroscopic binary stars according to Simbad. These stars with unreliable or variable RVs were rejected.

Table 1 gives the number of cluster members retrieved in each of the catalogues after filtering, with the median uncertainty of the corresponding RVs, as quoted in the catalogues. Soubiran et al. (2018b) reported that the uncertainty corresponds to the standard error of the weighted mean for stable stars that were followed-up for exoplanet detection. The high precision of individual measurements and the number of observations explain the very low median uncertainty of that catalogue $\left(0.002 \mathrm{~km} \mathrm{~s}^{-1}\right)$. APOGEE, OCCASO, and the catalogues from Mermilliod et al. $(2009,2008)$ and Nordström et al. (2004) also contain stars that have been observed several times in order to identify binaries. In 

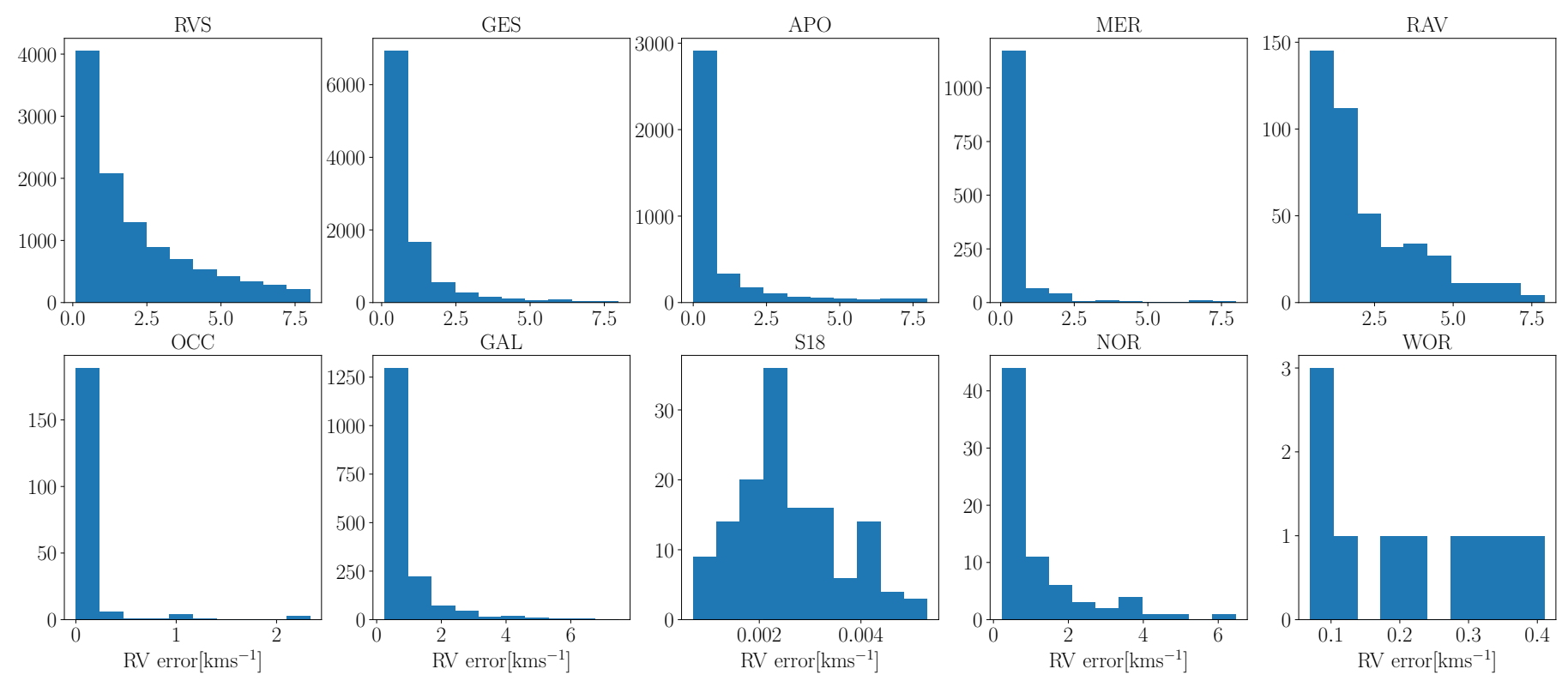

Fig. 1. Distribution of the RV uncertainties of each catalogue, designated by short names as listed in Table 1.

that case, the RV uncertainty corresponds to the quadratic sum of the single measurement error with the scatter of the measurements. RAVE also has multiple observations for a small fraction of stars and provides the individual values with their uncertainty corresponding to the error of a single measurement. Finally, the Gaia-ESO survey includes RVs of the same star obtained with different setups that give different measurement errors (Jackson et al. 2015).

This star sample is dominated by dwarfs and has a median effective temperature Teff of $4874 \mathrm{~K}$ (based on Teff provided in Gaia DR2); more than $13 \%$ of the stars are hotter than $6250 \mathrm{~K}$. We note that the uncertainties of stars hotter than $6250 \mathrm{~K}$ are significantly larger (median $1.75 \mathrm{~km} \mathrm{~s}^{-1}$ ) than those of cooler stars (median $0.53 \mathrm{~km} \mathrm{~s}^{-1}$ ). This mainly reflects the lower precision of the RVs of rotating stars, which are frequent among AF-type stars in young OCs. Figure 1 shows the distribution of the RV uncertainties of each catalogue. The uncertainties are not determined in the same way in the different catalogues. The 28371 RV measurements of OCs members that we gathered correspond to 23453 different stars.

\subsection{Zero-points of RV catalogues}

This sample allowed us to assess the consistency of RVs in the different catalogues. The comparison of RVs for stars in common in two catalogues gives an idea of potential offsets due to zero-point differences, together with their typical precision. Zero-point differences are a result of the different observing modes, instrumental characteristics, and calibration procedures of each instrument. They have to be taken into account when $\mathrm{RV}$ s of different origins are combined. A subset of 3116 stars has measurements in two or more catalogues. The RV difference between catalogues that have more than 20 stars in common is presented in Fig. 2, and the corresponding comparison of catalogues is listed in Table 2. The RVs agree well in general, with offsets smaller than $0.5 \mathrm{~km} \mathrm{~s}^{-1}$ and between 0.5 and $1.4 \mathrm{~km} \mathrm{~s}^{-1}$ for comparisons that involve RAVE or GALAH. The dispersions (measured by the median absolute deviation MAD) are typically about $1 \mathrm{~km} \mathrm{~s}^{-1}$ or lower, consistent with the precision of the catalogues listed in Table 1 for all combinations of surveys. In these comparisons of the different catalogues, we did not see any significant trend with colour or apparent magnitude.

Most of the RVs available for the cluster members are provided by Gaia DR2 or the Gaia ESO survey. A large fraction of the GES observing program is dedicated to OCs and nicely increases the number of clusters for which an RV can be computed. Gaia and GES have an offset of $\sim 0.3 \mathrm{~km} \mathrm{~s}^{-1}$, similar to the offset between GES and APOGEE, and Gaia and APOGEE agree at a level better than $0.1 \mathrm{~km} \mathrm{~s}^{-1}$.

The zero-point of RAVE is found to be different from that of the other surveys by $\sim 1 \mathrm{~km} \mathrm{~s}^{-1}$ (RAVE underestimates the RVs compared to the others), which is larger than reported in previous studies. Steinmetz et al. $(2018,2020)$ and Sartoretti et al. (2018) reported an offset of $\sim 0.3 \mathrm{~km} \mathrm{~s}^{-1}$ between RAVE and Gaia DR2. Even when we applied strong quality cuts on both RAVE and Gaia DR2, the median difference remained $1 \mathrm{~km} \mathrm{~s}^{-1}$. RAVE also differs from APOGEE by $1.42 \mathrm{~km} \mathrm{~s}^{-1}$, and from Mermilliod et al. $(2008,2009)$ by $0.79 \mathrm{~km} \mathrm{~s}^{-1}$. The agreement with $\mathrm{S} 18$ is better at a level of $0.2 \mathrm{~km} \mathrm{~s}^{-1}$, however, which might be related to the bright magnitude of the common stars and their colour range. We also note a systematic offset of GALAH DR3 with all the other catalogues, opposite to that of RAVE and of smaller amplitude, but still larger than the value of $0.22 \mathrm{~km} \mathrm{~s}^{-1}$ reported by Buder et al. (2020) for the comparison to Gaia DR2. These larger offsets might be related to the fact that our star sample is dominated by dwarfs, with a fraction of early-type stars larger than in RAVE and GALAH in general. For RAVE, we see indeed a trend that the offset is slightly larger for hot stars than for cool stars. In order to set all the RVs on the scale of Gaia, we applied a zero-point correction to the individual RVs from all the non-Gaia catalogues, according to the offsets listed in Table 2.

\subsection{Mean RVs}

In order to compute the mean RV of each OC, we first computed the mean RV of each star, because some of them (12\%) have multiple measurements from different catalogues. For both the mean per star and the mean per cluster, we used a weighted procedure based on the errors of individual measurements, following Soubiran et al. (2013, 2018a). For each star or each cluster, 


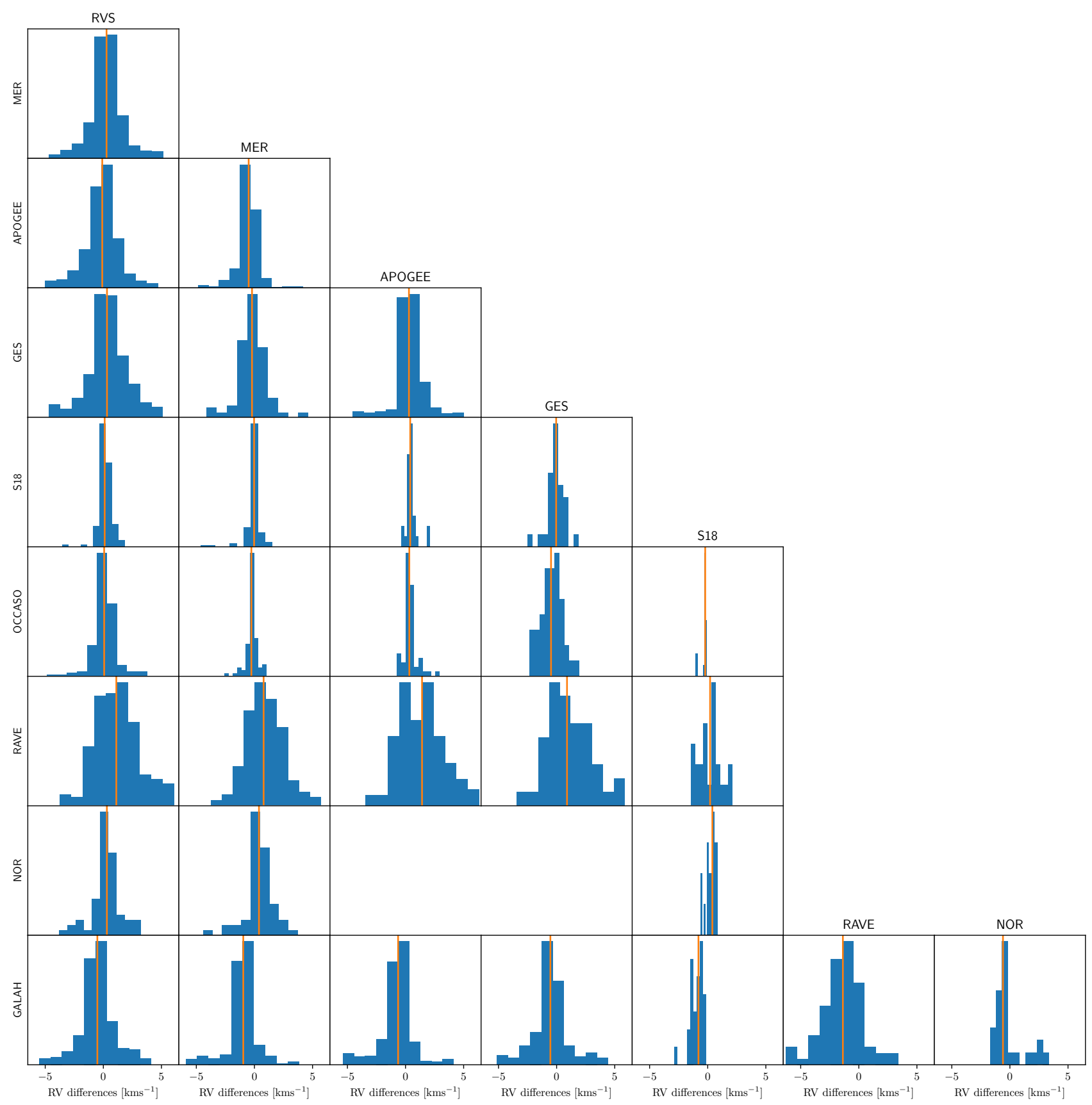

Fig. 2. Histograms of the RV difference of stars in common in several surveys (zoomed in the range from -5 to $5 \mathrm{~km} \mathrm{~s}^{-1}$ ). The solid orange line corresponds to the median value. The statistics of the comparisons are given in Table 2.

the mean RV was computed by attributing to each RV measurement a weight $\omega_{i}$ defined as $\omega_{i}=1 / \epsilon_{i}^{2}$, where $\epsilon_{i}$ is the RV error. Outliers were rejected on the basis of a $3 \sigma$ clipping. A fraction of about $8 \%$ of the stars with multiple measurements have an RV uncertainty larger than $3 \mathrm{~km} \mathrm{~s}^{-1}$, and we suspect that they are binaries with variable RVs. These stars were not rejected, but the procedure gives them less weight when the mean RV of their parent cluster is computed. However, in the large majority of cases, we have only one measurement per star so that binaries cannot be identified. Binaries that have large variations in RV may alter the mean RV of the parent OC when there are only a few members.

The catalogue of stars provides the mean RV in the Gaia RVS scale, with its uncertainty and the number of measurements, as well as the membership probability from Cantat-Gaudin et al. (2020). It includes 23424 unique stars, 97 of which appear twice owing to a non-null probability to belong to two different clusters that are located close to each other on the sky.

In order to compute the mean RV per OC, we considered only the stars with a membership probability higher than 0.4 . This is the threshold value found by Soubiran et al. (2018a) to be the best compromise between the largest number of members and the lowest contamination by field stars.

In the end, 1382 OCs have a mean RV, which represents an improvement by $60 \%$ compared to the previous RV catalogue of OCs that was based on Gaia only (Soubiran et al. 2018a). It also supersedes the catalogue by Carrera et al. (2019) that was based on Gaia, GALAH, and APOGEE. Before Gaia DR2, the two 
Table 2. Median (MED) and MAD of RV differences for stars observed by two surveys with the number of stars in common.

\begin{tabular}{lccc}
\hline \hline Catalogues & $N_{\text {stars }}$ & MED & MAD \\
\hline RVS - MER & 1079 & 0.28 & 0.68 \\
RVS - APO & 784 & -0.09 & 0.86 \\
RVS - GES & 486 & 0.31 & 1.06 \\
RVS - S18 & 115 & 0.12 & 0.26 \\
RVS - OCC & 161 & 0.07 & 0.40 \\
RVS - RAV & 299 & 1.12 & 1.36 \\
RVS - GAL & 607 & -0.51 & 0.88 \\
RVS - NOR & 65 & 0.31 & 0.49 \\
MER - APO & 304 & -0.50 & 0.33 \\
MER - GES & 152 & -0.21 & 0.72 \\
MER - S18 & 106 & -0.03 & 0.16 \\
MER - OCC & 82 & -0.26 & 0.17 \\
MER - RAV & 198 & 0.79 & 1.13 \\
MER - GAL & 293 & -0.96 & 0.49 \\
MER - NOR & 63 & 0.39 & 0.47 \\
APO - GES & 358 & 0.29 & 0.43 \\
APO-S18 & 30 & 0.40 & 0.10 \\
APO - OCC & 65 & 0.33 & 0.13 \\
APO - RAV & 125 & 1.42 & 1.60 \\
APO - GAL & 534 & -0.64 & 0.59 \\
GES - S18 & 30 & -0.04 & 0.41 \\
GES - OCC & 41 & -0.48 & 0.66 \\
GES - RAV & 57 & 0.89 & 1.89 \\
GES - GAL & 281 & -0.54 & 0.78 \\
S18 - OCC & 21 & -0.22 & 0.04 \\
S18 - RAV & 24 & 0.20 & 0.71 \\
S18 - GAL & 29 & -0.80 & 0.36 \\
S18 - NOR & 20 & 0.40 & 0.23 \\
RAV - GAL & 97 & -1.37 & 1.43 \\
GAL - NOR & 28 & 0.60 & 0.27 \\
\hline
\end{tabular}

Notes. Short names of the catalogues are the same as in Table 1.

largest compilations of cluster RVs were those of Kharchenko et al. (2013) and Dias et al. (2002), with 962 and 703 objects, respectively.

This is the first determination of the mean RV for most of the recently discovered OCs. In particular, half of the UBC clusters (Castro-Ginard et al. 2020) are part of our catalogue, and they represent nearly $20 \%$ of the full sample of OCs with a known RV. Of the 75 high-confidence clusters recently discovered by Liu \& Pang (2019), 35 are present in the latest catalogue from Cantat-Gaudin et al. (2020), and we provide an RV for most of them. For instance, we provide the mean RV of UBC 274 (also reported as LP 5), a recently discovered disrupting OC (CastroGinard et al. 2020; Piatti 2020), based on 18 members observed with RAVE, GALAH, and Gaia RVS (Fig. 3). By combining Gaia RVS and GES, several OCs now have RVs that are based on hundreds of members, the top two are Trumpler 5 and NGC 3532 with 659 and 664 stars, respectively. For about $18 \%$ of the sample, the mean RV is based on more than 10 stars, and for $50 \%$ it is based on at least 3 stars. The median uncertainty of the weighted mean $\mathrm{RV}$ is $1.13 \mathrm{~km} \mathrm{~s}^{-1}$ when the full sample is considered.

Of the clusters with fewer members, some exhibit a large error that renders their mean RV uncertain. The RVs of 430 OCs are based on only one star, with no information on its potential variability (binarity). This represents $31 \%$ of our sample, nearly the same proportion as in Soubiran et al. (2018a). Selecting
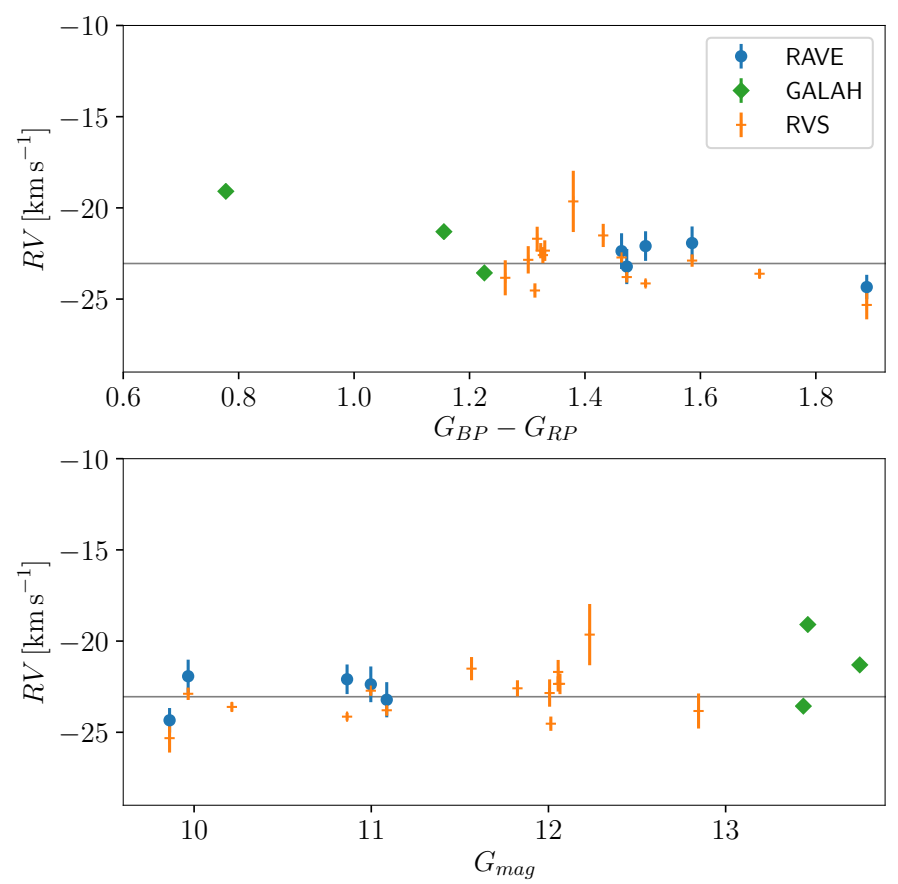

Fig. 3. Individual RV of UBC 274 members (proba $\geq 0.4$ ) from RAVE in blue, from GALAH in green, both after the zero-point correction indicated in Table 2, and from Gaia RVS in orange, as a function of the $G_{B P}-G_{R P}$ colour and $G$ magnitude from Gaia. The grey line represents the weighted mean, $\mathrm{RV}=-23.05 \pm 0.25 \mathrm{~km} \mathrm{~s}^{-1}$, based on 18 members.

the most reliable OCs that have an RV uncertainty lower than $3 \mathrm{~km} \mathrm{~s}^{-1}$ based on at least three stars, we obtain 513 clusters. This sub-sample has a median uncertainty of $0.55 \mathrm{~km} \mathrm{~s}^{-1}$ and a median number of nine stars. This means that we have 107 more reliable OCs than in Soubiran et al. (2018a).

The catalogue includes 21 high-velocity OCs with $|\mathrm{RV}|>100 \mathrm{~km} \mathrm{~s}^{-1}$, but 13 are based on a single star and are therefore to be considered with caution. The 8 remaining high-velocity clusters with more members were known before.

The catalogue of OC velocities (available at the CDS) provides the RV per cluster with its uncertainty and the number of members used on average. It also lists Galactic velocities, orbital parameters, and the actions computed in the next sections.

\section{Open clusters in phase space}

In this section, we combine the mean OC positions, distances, proper motions from Cantat-Gaudin et al. (2020), and our mean RVs to compute heliocentric and Galactocentric Cartesian and cylindrical positions and velocities for the full sample of 1382 OCs. For positions, the same conventions and reference values as Gaia Collaboration (2018d) were used: in Cartesian Galactocentric coordinates, the Sun is located at $X=-8.34 \mathrm{kpc}$, $Y=0 \mathrm{pc}$, and $Z=27 \mathrm{pc}$ from the centre of the Galaxy. Similarly, we set the azimuthal velocity $V_{c}$ at the solar radius at $240 \mathrm{~km} \mathrm{~s}^{-1}$. The velocity of the Sun with respect to the local standard of rest (LSR) is set to $(U, V, W)=(11.1,12.24,7.25) \mathrm{km} \mathrm{s}^{-1}$ (Schönrich et al. 2010). To calculate the uncertainties in the velocity space, we used a Monte Carlo sampling of the astrometric and RV measurements and their uncertainties, which we assumed to be Gaussian. In the following, we consider the standard deviations of the Monte Carlo samples as the uncertainties. We then obtain the full sample of OCs with median standard 

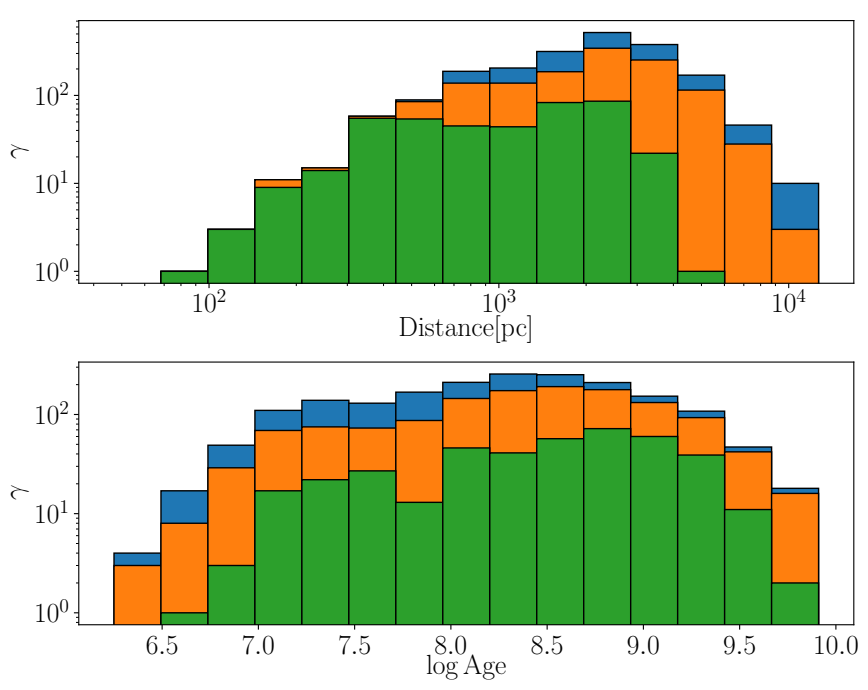

Fig. 4. Histogram in log scale of the heliocentric distance (top panel) and of the age distribution (bottom panel) for the full sample of OCs from Cantat-Gaudin et al. (2020) in blue, the sub-sample of OCs for which we have a RV measurement in orange, and the HQS in green.

deviations in the cylindrical velocities of $\left(\delta V_{r}, \delta V_{\phi}, \delta V_{z}\right)=$ $(2.8,3.2,1.6) \mathrm{km} \mathrm{s}^{-1}$.

For distant OCs, the astrometric uncertainties translate into large Galactic velocity errors. The standard deviations for 129 OCs on one of the velocity components are higher than $100 \mathrm{~km} \mathrm{~s}^{-1}$, all of them at distances from the Sun larger than $2.2 \mathrm{kpc}$. The most extreme cases are NGC 3105 and SAI 109, which have a relative parallax error of 82 and $99 \%$, respectively, and are both located at $6.9 \mathrm{kpc}$. For these two clusters, the velocity uncertainties reach several thousand $\mathrm{km} \mathrm{s}^{-1}$ in $V_{\phi}$ and $V_{z}$. Moreover, as mentioned in the previous section, some OCs do not have a fully reliable mean RV because they only have a few members, lack information about their stability, or because the dispersion among the members is large.

We therefore defined a high-quality sample (HQS) composed of the OCs with a reliable mean RV (uncertainty lower than $3 \mathrm{~km} \mathrm{~s}^{-1}$ based on at least three members as defined in the previous section) and with standard deviations on $\left(V_{r}, V_{\phi}, V_{z}\right)$ below $10 \mathrm{~km} \mathrm{~s}^{-1}$. This HQS is composed of 418 OCs with median uncertainties on $\left(V_{r}, V_{\phi}, V_{z}\right)$ of $(1.2,1.3,1.0) \mathrm{km} \mathrm{s}^{-1}$. This is very similar to the median uncertainty of the field star sample selected by Gaia Collaboration (2018d), who quoted $\left(\delta V_{r}, \delta V_{\phi}, \delta V_{z}\right)=$ $(1.2,1.3,1.0) \mathrm{km} \mathrm{s}^{-1}$ for stars with $\varpi / \sigma_{\varpi}>5$. For 411 out of these 418 clusters, a homogeneous estimation of their ages is provided by Cantat-Gaudin et al. (2020).

Figure 4 shows the histogram of the heliocentric distance and logarithmic ages of our different samples. Our subsample of 1382 OCs with RV measurements is $90 \%$ complete with respect to the total sample of 2017 OCs from Cantat-Gaudin et al. (2020) up to $860 \mathrm{pc}$, and our HQS is $90 \%$ complete with respect with the total sample up to $500 \mathrm{pc}$.

The bottom panel shows that except for the very young clusters, all ages are represented in the HQS, which indicates that our selection does not introduce a noticeable bias in terms of the age distribution for clusters older than $10 \mathrm{Myr}$. The kinematical selection based on the RV uncertainty removed most of the very young clusters because the RV of young stars is less reliable.

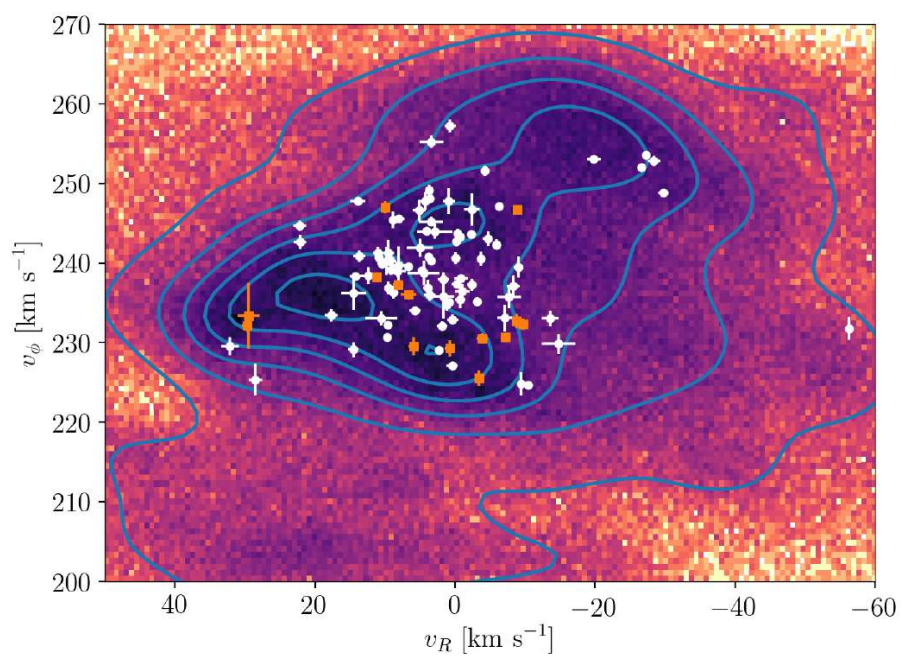

Fig. 5. $\left(V_{\phi}, V_{r}\right)$ distribution of field stars in the solar neighbourhood (closer than $200 \mathrm{pc}$ ) compared with clusters closer than $500 \mathrm{pc}$ (white) and $200 \mathrm{pc}$ (orange).

\subsection{Kinematics of OCs compared with field stars}

In this section, we compare the kinematics of the field stars sample from Gaia Collaboration (2018d) with that of the OC sample. In Fig. 5 we compare the $\left(V_{\phi}, V_{r}\right)$ distribution of the nearby OCs within $500 \mathrm{pc}$ and $200 \mathrm{pc}$, with the field stars from Gaia DR2 closer than $200 \mathrm{pc}$ and having a relative error in parallax $<0.05$. In this space, field stars from the solar neighbourhood show clear substructures in form of arches and clumps, which are associated with resonances due to non-axisymmetric features of the Galaxy, as discussed in Gaia Collaboration (2018d) and subsequent works. Large clumps were found to be associated with previously known moving groups (Eggen 1958; Dehnen \& Binney 1998; Chereul et al. 1999; Nordström et al. 2004), and several overdensities of field stars in this space have been linked with OCs before (Gaia Collaboration 2018d). The general trend of our sample of clusters closer than $500 \mathrm{pc}$ is to overlap with the higher density regions of field stars, as previously reported in Soubiran et al. (2018a). The several OCs that we obtained here fall in the central part of the velocity distribution. Although we do not see a clear clumping in the cluster distribution, OCs appear to be most frequent in a band just between the two central arches of the field stars. This shows that OCs do not follow the exact distribution of overdensities drawn by the field stars. This was previously reported based on a smaller number of clusters in Gaia Collaboration (2018d), who asserted that only the Pleiades and Hyades clusters are associated with overdensities, and that other clusters do no show a particular overdensity in this space.

In Fig. 6 we plot the distribution of azimuthal velocities as a function of Galactocentric radius of the full sample of OCs and the HQS. Field stars in this figure were retrieved from the Gaia archive by selecting stars with the same criteria as Gaia Collaboration (2018d), providing a sample of 6376803 stars. In this space, Antoja et al. (2018) showed that the diagonal ridges can be signatures of phase-mixing after a perturbing event, or alternatively, can be due to resonances of a barred potential or of the spiral arms. Kawata et al. (2018) argued that many of the diagonal ridges are likely related with the perturbations from the bar outer Lindblad resonance and spiral arms, which may be explained with the transient spiral arm scenario. Dias et al. (2019) and Barros et al. (2020) claimed that spiral resonances are able to trap stars or OCs orbits inside the corotation radius 


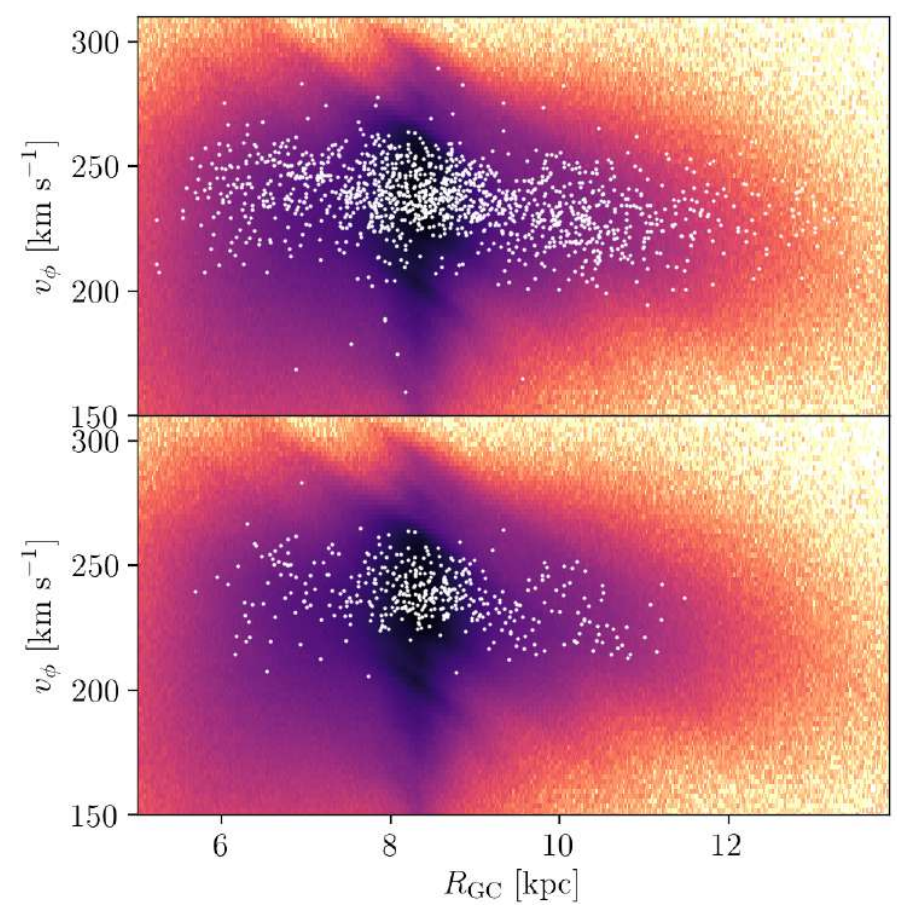

Fig. 6. $\left(V_{\phi}, R_{\mathrm{GC}}\right)$ distribution of field stars coloured by density, compared with clusters in white. In the top panel we plot the full sample of clusters, and in the bottom panel we show the HQS.

and associated the corotation with some ridges. In our plot, the clusters seem to qualitatively follow the distribution of diagonal overdensities described by the ridges, at least the most prominent ones. The recent $N$-body simulations by Khanna et al. (2019) suggest that the ridges are more prominent for stars close to the plane with solar metallicity; these conditions are generally met by a sample of OCs. Probably because of the shape of these ridges, the velocity gradient of $V_{\phi}$ decreases towards large radii, which is more clearly visible in the plot that shows the entire cluster sample. This is consistent with the top middle panel of Fig. 9, where outer clusters (yellow) have lower velocities (and in general have older ages, see the histogram). As discussed in more detail in the next subsection, this produces a dip in the rotation curve that is traced by our sample of OCs.

\subsection{Rotation curve of the Milky Way}

We represent in Fig. 7 the azimuthal velocities of the full sample and the HQS OCs as a function of the Galactocentric radius, superimposing a theoretical rotation curve extracted from the axisymmetric gravitational potential described in Sect. 4. Our sample of OC data describes the rotation curve of the Milky Way for a mostly young population in the range $R_{\mathrm{GC}} \sim[6.5,10.5] \mathrm{kpc}$ with good precision. In the bottom panels we represent the median value of the rotation velocity in bins of approximately $400 \mathrm{pc}$. Uncertainties are computed as $\sqrt{\pi / 2} \cdot \sigma / \sqrt{N}$. We only plot the points computed using more than $20 \mathrm{OCs}$, which we consider to be reliable estimates and not dominated by outliers.

The HQS and the full sample show a similar behaviour. The samples overlap with what is expected from the axisymmetric curve ("MWPotential2014"). Several bins in both samples depart from the theoretical curve and show a small dip towards the inner Galaxy $\left(R_{\mathrm{GC}} \sim 7 \mathrm{kpc}\right)$, and a significant dip at $R_{\mathrm{GC}} \sim 9.7 \mathrm{kpc}$, which departs by more than $2 \sigma$ from the theoretical curve. In the full sample, the outer dip extends to $11 \mathrm{kpc}$.
Gaia Collaboration (2018d) reported similar dips in the sample of small $Z$ (their Fig. 13), but the inner dip is only seen at positive azimuths. In the bottom panels, we overplot the unified rotation curve computed by Sofue (2020), which was obtained from a combination of data from different sources in the literature. Interestingly, these data show a significant drop around $10 \mathrm{kpc}$, although with a different depth than our values.

A dip in the region $R_{\mathrm{GC}} \sim 9.5 \mathrm{kpc}$ has been reported variously before (Reid et al. 2019; Barros et al. 2016; Sofue et al. 2009), but its nature is not fully understood. It has been tentatively explained by a ring density structure observed in the neutral $\mathrm{H}$ gas slightly farther out than the solar radius $\left(R_{\mathrm{GC}} \sim 10 \mathrm{kpc}\right.$ Nakanishi \& Sofue 2016), which successfully reproduces the dip. Dias et al. (2019) calculated a corotation radius of $8.51 \pm$ $0.64 \mathrm{kpc}$ and found a similar dip in their rotation curve, which can therefore be attributed to the effect of corotation. Clusters located outside the corotation radius have a slower azimuthal velocity than clusters located inside. The depth of the dip is different depending on the data used, the dip we observe here is compatible with the recent study by Reid et al. (2019) from starforming regions: a small decrease from the model of $\sim 5 \mathrm{~km} \mathrm{~s}^{-1}$. Moreover, the general decreasing slope in the outer Galaxy shown by our data is also seen in the star-forming regions. Other studies from Cepheids (e.g., Mróz et al. 2019) also showed a slight decrease in $V_{\phi}$ before and after the solar radius. McGaugh (2019) built a model that reconciled the observed stellar rotation curve with the curve seen by interstellar gas, taking the overdensities of the spiral arms into account. In their Fig. 4, the modelled points (green) show a clear dip inside the solar radius that is compatible with the dip we see in the cluster data in Fig. 7.

Compared with Fig. 6, these dips are a direct consequence of the shape of the most prominent diagonal ridges, which are clearly shown by field stars in the Gaia RVS, but also by our clusters, where a remarkable decreasing velocity gradient is seen towards the outer Galaxy. This effect was analysed in MartinezMedina et al. (2019) using a non-axisymmetric Galactic model that showed that the two bar-spiral arms produce diagonal-like ridges of stars of constant angular momentum. They showed that these structures tend to clump the stars in the $V_{\phi}-R_{\mathrm{GC}}$ plane, pulling them up at the beginning of the ridge, and down at the end. This translates into wiggles in the rotation curve of the Galaxy. We overplot the rotation curve derived from the model by Martinez-Medina et al. (2019) ${ }^{1}$ in Fig. 7. Interestingly, the model resembles the two dips we observed in the OC data. We attribute this to the same phenomenon as the dips we observe in the cluster data, thus explained by the effect of non-axisymmetric structures in the angular momentum of the stars.

\subsection{Age dependence of Galactic velocities}

Figure 8 shows the distribution of the HQS in the $\left(V_{r}, V_{\phi}\right)$, $\left(V_{r}, V_{Z}\right)$, and $\left(V_{\phi}, V_{Z}\right)$ planes coloured by age. Several distant old OCs with extreme velocities $\left(V_{r}>40 \mathrm{~km} \mathrm{~s}^{-1}\right)$ have recently been discovered. Those included in the HQS are LP 930, UBC 326 , and UBC 324, which are all located farther than $1 \mathrm{kpc}$ in heliocentric distance. The most extreme OCs in total velocity $\left(\sqrt{V_{r}^{2}+V_{\phi}^{2}+V_{z}^{2}}\right)$ are Ruprecht 171, Haffner 5, and Berkeley 32, which are known as high-velocity objects for the disc.

\footnotetext{
1 We have scaled the rotation curve of the model to fit the reference frame used in our work, described in Sect. 3, using the expression $V_{\text {new }}=V_{\text {old }}+\frac{R_{\odot}}{8.5}\left(\theta_{\odot}-214\right)$, which was also used in Mróz et al. (2019).
} 


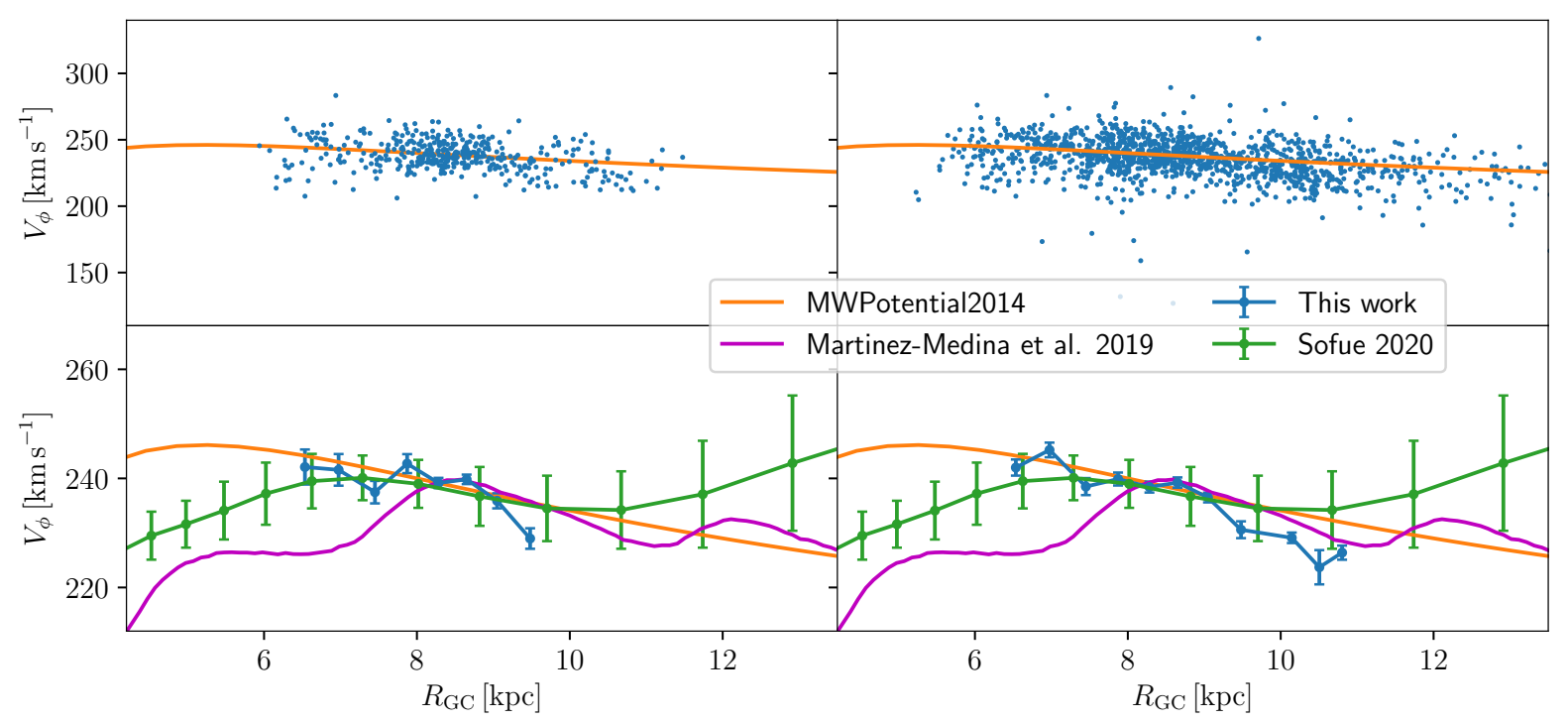

Fig. 7. Top: azimuthal velocities of all the OCs in the full sample (right) and the HQS (left) as a function of $R_{\mathrm{GC}}$. The rotation curve of the gravitational potential in Sect. 4 is superimposed. Bottom: median azimuthal velocities in bins of $R_{\mathrm{GC}}$. We also superimpose the unified rotation curve computed by Sofue (2020) and the non-axisymmetric model by Martinez-Medina et al. (2019).

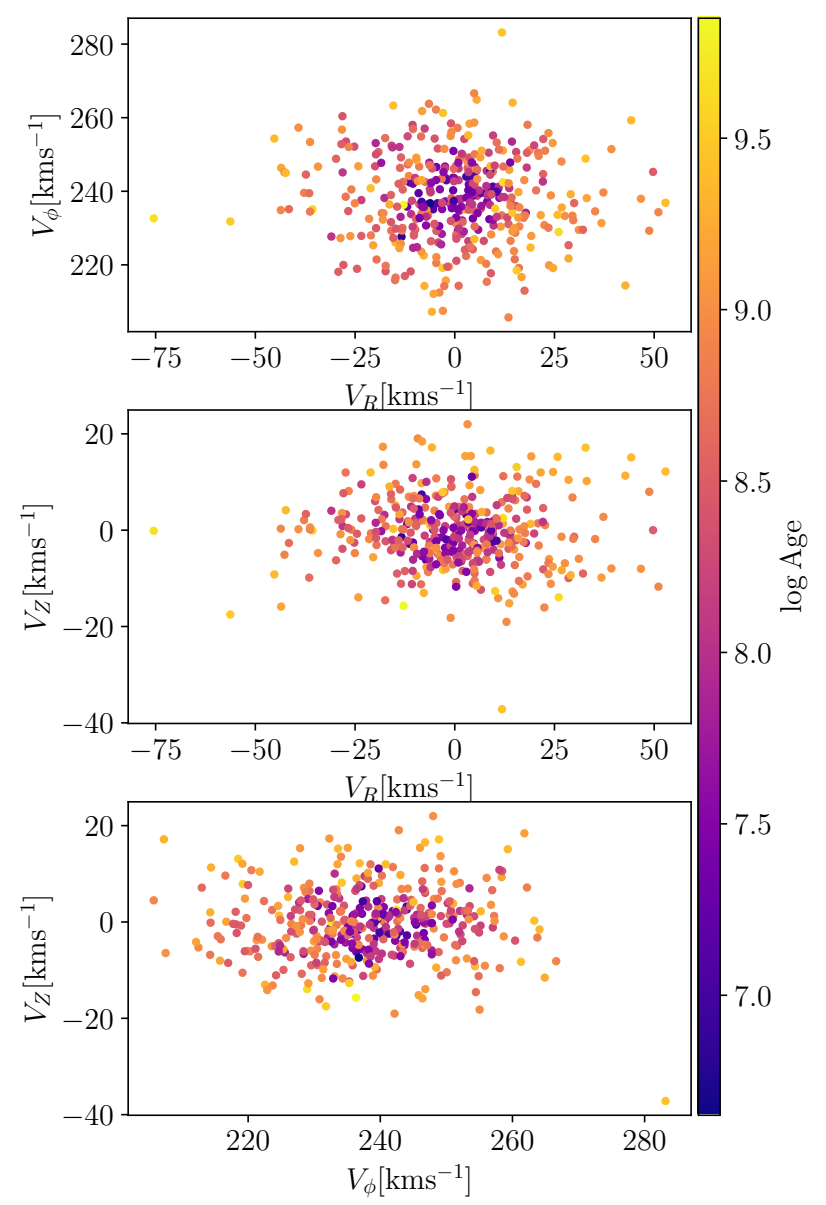

Fig. 8. Top: distribution of the HQS in Galactic velocities $\left(V_{r}, V_{\phi}\right)$ with colour related to age. Middle: same with the velocities $\left(V_{r}, V_{Z}\right)$. Bottom: same with the velocities $\left(V_{\phi}, V_{Z}\right)$.

The three components of the velocities of the HQS clusters as a function of age are shown in the top panel of Fig. 9. Clearly, they show a significant growing spread as age increases, which is related with the kinematic heating and is discussed in more detail in Sect 3.4. The radial and vertical velocities are centred on zero, and the azimuthal velocity is centred on the azimuthal velocity of the LSR. In addition, the middle panel of the top row shows that the azimuthal velocity of the oldest clusters within a given age range decreases when the radius increases. This trend is only noticeable for old clusters. Our kinematical selection based on the RV quality and errors of the Galactic velocities removed most of the young distant clusters of our sample. This bias was previously highlighted by Gaia Collaboration (2018d): $\mathrm{RVs}$ for young stars are often less reliable because their temperature is high, because they rotate, or because they are closer to the Galactic plane and cannot be observed spectroscopically because of heavy extinction. Inner clusters, moreover, (in blue) are preferentially old, with a wide range of azimuthal velocities, a dozen of them have $V_{\phi}<220 \mathrm{~km} \mathrm{~s}^{-1}$. For the vertical velocity shown in the right panel of the top row, the increase in dispersion highlights the fact that the clusters that reach higher $V_{Z}$ are in general the oldest ones.

The bottom panels of Fig. 9 show the histograms of each component of the velocities in several age bins. The youngest clusters form a quite symmetric distribution, centred near zero for the radial and vertical components (median value of $V_{r}=$ $-0.3 \mathrm{~km} \mathrm{~s}^{-1}$ and $V_{Z}=-1.3 \mathrm{~km} \mathrm{~s}^{-1}$ ) and near the azimuthal velocity of the LSR for the azimuthal component (median value of $V_{\phi}=239.5 \mathrm{~km} \mathrm{~s}^{-1}$ ). In the radial component, as age increases, the distribution becomes less Gaussian and the peak of the histogram tends to move towards larger $V_{r}$, although this is dependent on the binning of the histogram and the age limits that were assumed. Also depending on the binning, there seems to be a sign of bimodality in the four older age bins, although low number statistics does not allow us to reach a significant conclusion. However, regardless of the binning, the oldest bin has a significantly higher maximum value (around $\sim 14 \mathrm{~km} \mathrm{~s}^{-1}$ ) than the two youngest ones. As age increases, the distribution becomes more and more asymmetric, with a large tail towards negative $V_{r}$, and a smaller tail on the positive side becoming more populated at older ages. This effect is also independent of the histogram binning and age limits. This implies that clusters moving inwards do it with a wider range of radial motions than those moving 

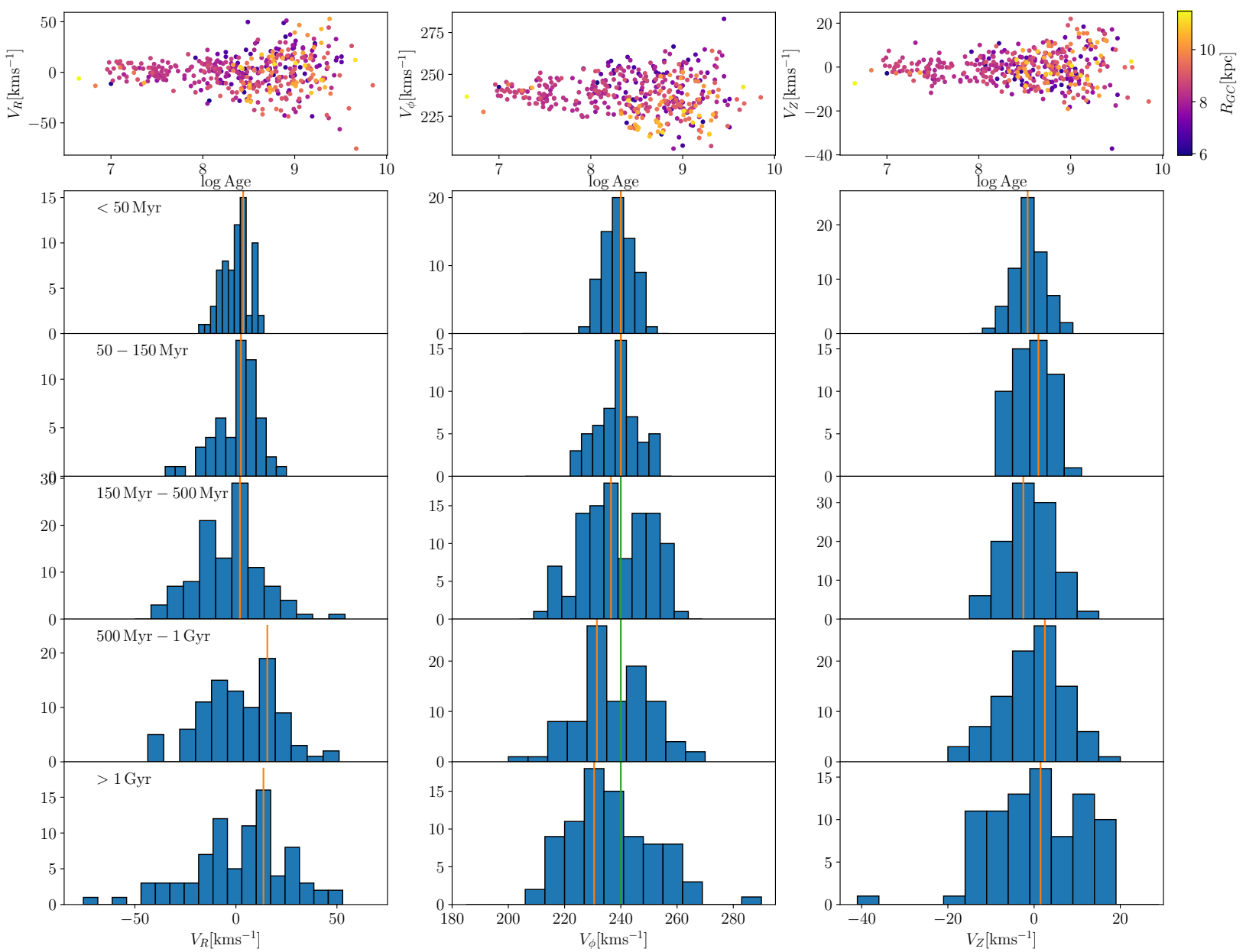

Fig. 9. Top: radial, azimuthal, and vertical velocities $V_{r}, V_{\phi}$, and $V_{Z}$ as a function of age for the HQS with the Galactocentric radius shown with colours as indicated in the colour bar. Bottom: distribution of $V_{r}, V_{\phi}$, and $V_{Z}$ of the OCs in different age bins. In each panel, the mode of the distribution is marked with a vertical orange line, and the azimuthal velocity of the LSR is marked with a vertical green line in the middle column.

outwards. This trend is noticeable in the whole range of ages represented in our OC population, which is representative of the youngest population in the Galactic disc. The majority of old clusters of our sample have a positive $V_{r}$, highlighting that OCs that move outwards have a higher probability of survival than clusters moving inwards. For the azimuthal component, the histograms in bins of ages clearly show the asymmetric drift: in the two youngest bins the distribution is centred nearly on $240 \mathrm{~km} \mathrm{~s}^{-1}$, and the mode of the histogram shifts towards lower values in the older bins. In the three oldest bin, the most probable value is around $230 \mathrm{~km} \mathrm{~s}^{-1}$. In the third and fourth age bins, we can also note a bimodality of the distribution that is likewise seen for the radial component of the velocity, the bimodality being independent of the binning here, however. As age increases, the growing spread of the vertical velocity is clearly visible in the histograms, particularly in the two last age bins, where the distribution becomes asymmetric but remains centred near zero.

Figure 10 shows the distribution of $V_{\phi}$ of the HQS sample in the $X-Y$ plane in four different age bins. The spiral arms modelled by Reid et al. (2014) are represented by the shaded structures, and the updated Cygnus arm from Reid et al. (2019) is represented by the dashed shaded structure. We note that in the two youngest bins, OCs are mainly located close to the Sun near the local arm, and this spatial distribution is very different from that of the original sample presented in Cantat-Gaudin et al. (2020; their Fig. 8). This is a consequence of the bias of our sample stated above. The remaining nearby young clusters show a remarkable homogeneity in azimuthal velocities, with a typical velocity dispersion of $5.5 \mathrm{~km} \mathrm{~s}^{-1}$ around solar velocity. In the two oldest age bins, OCs are much more spatially and kinematically dispersed and no longer follow the spiral arms.

\subsection{Age velocity relation for open clusters}

The increasing trend of the velocity dispersions for field stars with age is known as the age-velocity relation (AVR) and has been discussed for decades (e.g., Wielen 1977; Freeman 1987; Nordström et al. 2004). The AVR is reasonably described as a power law with some saturation at the oldest ages (Binney \& Tremaine 2008; Aumer \& Binney 2009; Soubiran et al. 2008), altough the shape of the relation is debated (Martig et al. 2014). The AVR involves different mechanisms, such as the radial migration and heating induced by giant molecular clouds, by the spiral arms (Mackereth et al. 2019), or by the bar (Grand et al. 2016), which affect the velocity components differently. The AVR study is complicated by the mixture of different stellar populations present in the solar neighbourhood, such as the thin and thick discs, which have different scale heights and different star formation and heating histories (Yu \& Liu 2018; Mackereth et al. 2019). Similarly, inner and outer populations are differently affected by the dynamical 


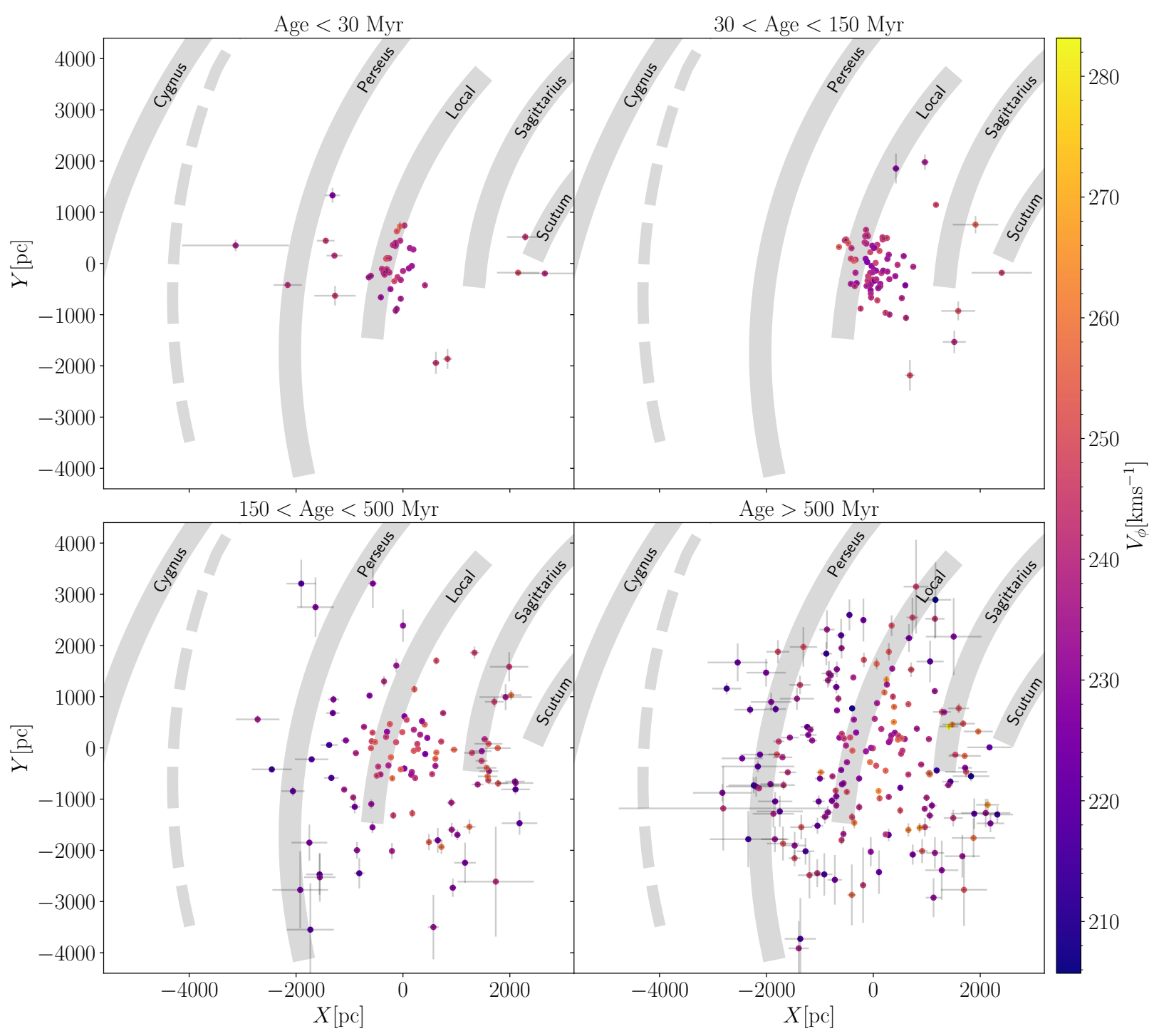

Fig. 10. Distribution of the HQS OCs in heliocentric Cartesian coordinates. Colours stand for the azimuthal velocity $V_{\phi}$. The shaded structures show the spiral arm modelled by Reid et al. (2014), and the dashed shaded structure represents the updated Cygnus arm from Reid et al. (2019).

effect of the bar, the spiral arms, and accretion events. In addition, uncertainties in the determination of stellar ages strongly affect the AVR (Aumer et al. 2016). Ages of OCs are more reliable than ages of individual stars and have been determined homogeneously in Cantat-Gaudin et al. (2020). This allowed us to investigate the AVR of OCs in a wide age range up to $\sim 2.5 \mathrm{Gyr}$ (too few clusters are older than this limit) and with a significant number of objects below $1 \mathrm{Gyr}$. The spatial extension is mostly confined within $200 \mathrm{pc}$ from the Galactic plane, so that the OC population is representative of the thin disc. It is thus interesting to compare the velocity ellipsoids of OCs and field stars in the age range in which they overlap.

Figure 11 shows the velocity dispersions measured in seven age bins for the 418 OCs from the HQS with an age determination. In each age bin, we fitted a Gaussian to each velocity component. Each point represented in Fig. 11 stands for these Gaussian standard deviations and their uncertainties. As expected, the dispersion of each component increases with age. The values and ratios are given in Table 3. The OC kinematics is characterised by a clear anisotropy in the three components, at all ages. The radial dispersion $\sigma_{R}$ is always significantly larger than $\sigma_{\phi}$, which is significantly larger than $\sigma_{z}$, as also seen in local field stars (e.g., Binney \& Tremaine 2008; Kuijken \& Tremaine 1994; Anguiano et al. 2018). The dispersion ratios remain globally stable in the different age bins, with some fluctuations within the error bars. This implies that the velocity ellipsoid maintains the same shape at all ages. Following previous studies of field stars, we fitted the increase in velocity dispersion with age $(\tau)$ in the form of a power law $\sigma_{V} \propto \tau^{\beta}$, as suggested in Sect. 8.4 of Binney \& Tremaine (2008) and by Jenkins (1992). We used a maximum likelihood (ML) estimator on the individual clusters of the HQS (ages younger than 2.5 Gyr), assuming Gaussian velocity errors. The results of the fits are shown in Fig 11. The grey lines represent the uncertainties on the fits: we show 100 fits taken from the final sample of the ML. The solid black line shows the best-fit power law obtained with an ML. This best fit is defined as the median value of the 16000 fits. We found $\beta_{R}=0.25_{-0.03}^{+0.05}, \beta_{\phi}=0.23_{-0.03}^{+0.03}$, and $\beta_{z}=0.19_{-0.03}^{+0.03}$. These values show that the heating rate of OCs is about the same in all directions, which agrees with the observation that the velocity ellipsoid maintains the same shape at all ages, as pointed out 


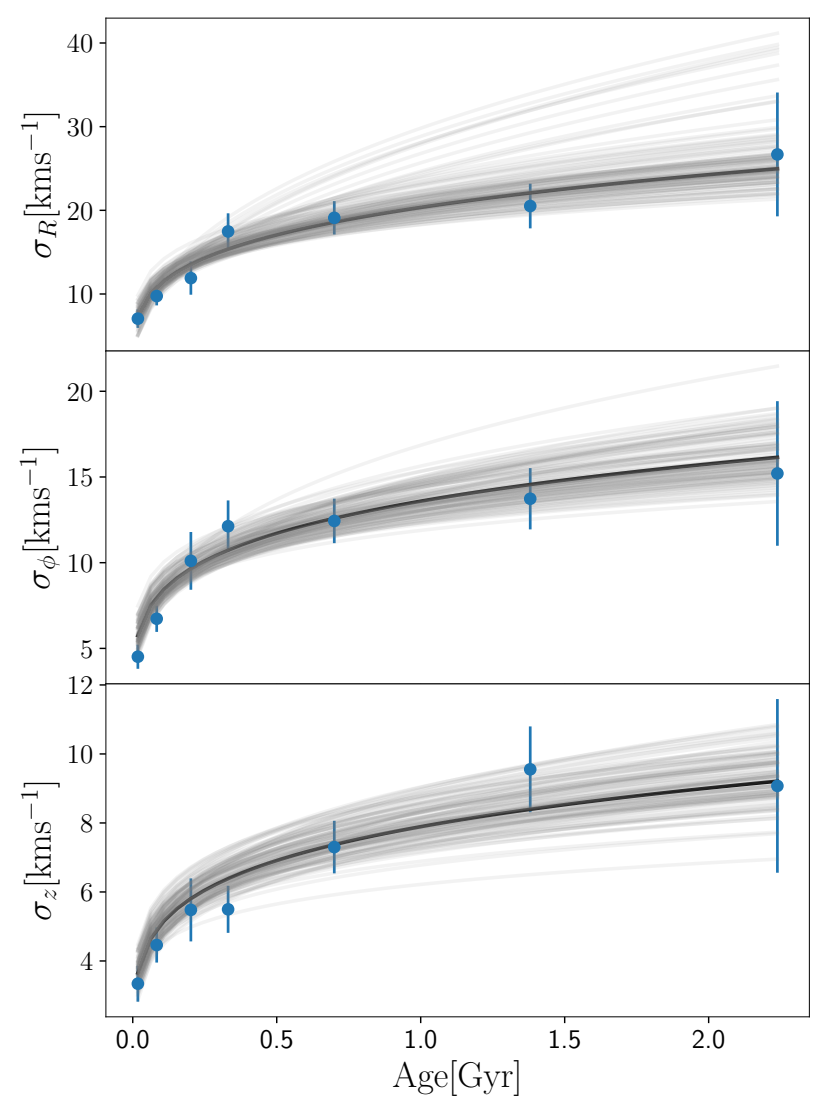

Fig. 11. Velocity dispersion of the cylindrical Galactocentric components $V_{r}, V_{\phi}$, and $V_{z}$ for the HQS. The blue dots and error bars stand for the standard deviations of the velocities and their uncertainties obtained by fitting a Gaussian on each component of the velocities in each of the seven age bins of Table 3, the oldest age bin having an upper limit of $2.5 \mathrm{Gyr}$. The $x$ value of the blue dots is the median value of the corresponding age bin. The black line shows the best-fit power law obtained using an ML on each cluster of the HQS younger than $2.5 \mathrm{Gyr}$, and the grey lines represent the uncertainties of the fits.

earlier. In the following, we compare this kinematical behaviour with that of field stars.

Yu \& Liu (2018) measured the AVR of $~ 3500$ local stars, splitting them into different subsamples depending on their $Z$ position and metallicity. Their metal-rich low- $Z$ sample is comparable to our OC sample, which is representative of the local thin disc. For their two youngest age bins, corresponding to $1.4 \pm 0.4$ and $1.9 \pm 0.3 \mathrm{Gyr}$, respectively, they found dispersions in the different components and dispersion ratios that agree well with what we found for OCs of similar age (see their Table 1). They fitted the heating parameters in the age range $1-8 \mathrm{Gyr}$ and determined $\beta_{R}=0.28 \pm 0.08, \beta_{\phi}=0.30 \pm 0.09$, and $\beta_{z}=0.54 \pm 0.13$, in agreement with our values in $R$ and $\phi$, but not in $Z$.

Mackereth et al. (2019) measured the heating parameters in $R$ and $Z$ with field stars with ages between 1 and 9 Gyr. They found $\beta_{z}=0.50$ for stars that were comparable to OCs in metallicity, which is higher than our value. They found that $\beta_{R}$ varies from 0.15 to 0.4 depending on the mean orbital radii. Recently, Sharma et al. (2020) assembled several large stellar samples that traced different populations from complementary large surveys and using different age estimators. They found consistent relations for all with $\beta_{R}=0.251 \pm 0.006$ and $\beta_{z}=0.441 \pm 0.007$, which again agrees with our value for $R$, but not $Z$. Mackereth et al. (2019) and Sharma et al. (2020) highlighted a complex relation between $\sigma_{R} / \sigma_{z}$ with age, depending on metallicity, angular momentum, and height above the plane.

These comparisons of the heating parameter $\beta$ (see Table 4 for more clarity) tend to show that the OC population has a dynamical evolution similar to the field stars in the radial and azimuthal directions, but not in the vertical direction. OCs seem to have a lower heating rate in $Z$ than field stars. The main difference between our determination of $\beta$ and that of Yu \& Liu (2018), Mackereth et al. (2019) and Sharma et al. (2020) is the age range which extends to young ages for OCs (half of the OCs are younger than 360 million years) with few objects older than $3 \mathrm{Gyr}$, while the stellar samples typically range between 1-10 Gyr. Although their age distributions overlap around $1-2 \mathrm{Gyr}$, the fit of the $\beta$ parameter is not performed in the same age range for OCs and field stars. It is therefore interesting that the heating rate is found to be similar in the Galactic plane but not perpendicular to the plane. This could mean that clusters do not reach high altitude and older ages because they are disrupted before, which therefore introduces a bias in our sample. The small $\beta_{z}$ could also reflect that giant molecular clouds, which are the main cause of the vertical scattering of field stars (Lacey 1984; Jenkins \& Binney 1990), are not as efficient in scattering OCs, or that the effect of the giant molecular clouds is to disrupt the OCs.

The heating of the Galactic disc and the destruction of clusters have been simulated by Gustafsson et al. (2016), among others. They found that the fraction of massive old OCs, scattered into orbits with $|Z|>400 \mathrm{pc}$, is typically $0.5 \%$. Of the full initial sample of 2017 OCs from Cantat-Gaudin et al. (2020), $4 \%$ have $|Z|>400 \mathrm{pc}$. They are mainly old, with a median age of $\sim 2 \mathrm{Gyr}$, and at Galactocentric distances ranging from 7.9 to $20 \mathrm{kpc}$, as previously reported by Cantat-Gaudin et al. (2020). However, after our quality cuts, only six such clusters remain in the HQS, making the statistics too poor to reach a conclusion on their origin.

\section{Actions and orbital parameters}

In this section we use the full 6D coordinates of the samples of clusters to compute orbits and action variables, and analyze them as a function of the age. We used the python package galpy (Bovy 2015) for Galactic dynamics to integrate the orbits and compute the action-angle variables.

We used the axisymmetric potential MWPotential2014 implemented in galpy, which was derived by Bovy (2015) by fitting a simple model to existing dynamical data of the Milky Way. It is composed of a bulge, a Miyamoto-Nagai disc (Miyamoto \& Nagai 1975), and a dark matter halo modelled with a Navarro-Frenk-White (NFW) potential (Navarro et al. 1997). For the sake of comparison, we also used the axisymmetric potential model from McMillan (2017) to confirm our results. This model was also fitted to the mass distribution of the Milky Way and consists of several components representing the cold gas disc close to the Galactic disc, both the thin and thick discs, a bulge, and a dark matter halo. The detailed parameters of each component of these potentials are listed in Bovy (2015) and McMillan (2017).

\subsection{Orbits}

We integrated each cluster orbit with an integration step of $0.01 \mathrm{Myr}$ up to $500 \mathrm{Myr}$. We did not attempt to integrate more 
Table 3. Dispersions of velocity components and ratios in the same bins as in Fig. 11.

\begin{tabular}{lccccccc}
\hline \hline Age interval (Gyr) & $N$ & $\sigma_{R}$ & $\sigma_{\phi}$ & $\sigma_{z}$ & $\sigma_{R} / \sigma_{\phi}$ & $\sigma_{R} / \sigma_{z}$ & $\sigma_{\phi} / \sigma_{z}$ \\
\hline Age $<0.03$ & 43 & $7.05 \pm 1.10$ & $4.52 \pm 0.71$ & $3.34 \pm 0.52$ & $1.56 \pm 0.49$ & $2.11 \pm 0.66$ & $1.35 \pm 0.42$ \\
$0.03-0.15$ & 79 & $9.75 \pm 1.11$ & $6.73 \pm 0.77$ & $4.46 \pm 0.51$ & $1.45 \pm 0.33$ & $2.19 \pm 0.50$ & $1.51 \pm 0.34$ \\
$0.15-0.25$ & 38 & $11.90 \pm 1.98$ & $10.11 \pm 1.68$ & $5.48 \pm 0.91$ & $1.18 \pm 0.39$ & $2.17 \pm 0.72$ & $1.84 \pm 0.61$ \\
$0.25-0.50$ & 67 & $17.48 \pm 2.17$ & $12.13 \pm 1.50$ & $5.50 \pm 0.68$ & $1.44 \pm 0.36$ & $3.18 \pm 0.79$ & $2.21 \pm 0.55$ \\
$0.50-1.00$ & 94 & $19.11 \pm 1.99$ & $12.44 \pm 1.30$ & $7.30 \pm 0.76$ & $1.54 \pm 0.32$ & $2.62 \pm 0.55$ & $1.70 \pm 0.36$ \\
$1.00-2.00$ & 61 & $20.51 \pm 2.67$ & $13.73 \pm 1.79$ & $9.55 \pm 1.24$ & $1.49 \pm 0.39$ & $2.15 \pm 0.56$ & $1.44 \pm 0.37$ \\
$2.00-2.50$ & 15 & $26.68 \pm 7.40$ & $15.21 \pm 4.22$ & $9.07 \pm 2.52$ & $1.75 \pm 0.97$ & $2.94 \pm 1.63$ & $1.68 \pm 0.93$ \\
\hline
\end{tabular}

Table 4. Comparison of the values of $\beta$ found here and in previous studies.

\begin{tabular}{lccc}
\hline \hline & $\beta_{R}$ & $\beta_{\phi}$ & $\beta_{z}$ \\
\hline This study & $0.25_{-0.03}^{+0.05}$ & $0.23_{-0.03}^{+0.03}$ & $0.19_{-0.03}^{+0.03}$ \\
Yu \& Liu (2018) & $0.28 \pm 0.08$ & $0.30 \pm 0.09$ & $0.54 \pm 0.13$ \\
Mackereth et al. (2019) & - & - & 0.50 \\
Sharma et al. (2020) & $0.251 \pm 0.006$ & - & $0.441 \pm 0.007$ \\
\hline
\end{tabular}

time (up to each cluster age) because the reliability of the results decreases significantly with time due to inaccuracies in the time dependence of the potential, amplification of the uncertainties in distance, and motions, among other effects (Gaia Collaboration 2018c). We computed uncertainties by integrating each orbit 1000 times with a Monte Carlo sampling in the same way as described in Sect. 3. Figure 12 shows the orbits of two OCs, as an example of the results we can obtain using galpy.

We extracted the orbital parameters of the 1315 OCs for which we have an age estimate to investigate their relation with age. We represent the evolution of the maximum altitude above the Galactic plane $\left(Z_{\max }\right)$ as a function of the age of the full sample of clusters and the HQS in the left panel of Fig. 13. In all the panels, the maximum height of clusters younger than $300 \mathrm{Myr}$ remains constrained close to the Galactic plane. This is shown more clearly in the bottom panel, where the running median of both samples increases only for an age higher than $1 \mathrm{Gyr}$. For both samples of OCs, the median age of the subsample of clusters that reach an altitude higher than $400 \mathrm{pc}$ is greater than 1.5 Gyr. The left panels of Fig. 13 also show the increase in dispersion of the maximum height of the OCs above the plane for ages older than 1 Gyr. This is usually attributed to the vertical heating of the disc: clusters are preferentially formed in the thin disc, and then giant molecular clouds and spiral arms tend to scatter them away from the midplane (Spitzer \& Schwarzschild 1951; Jenkins \& Binney 1990). This effect is consistent with what is shown in more detail in the right panels of Fig. 9, as we commented on in Sect. 3 .

The eccentricity as a function of age in the right panel of Fig. 13 shows several things. First, clusters younger than $30 \mathrm{Myr}$ $(\log ($ Age $) \sim 7.5)$ show very low eccentricity. This is best seen in the HQS sample where the maximum value in this range is 0.018 , while the full sample contains some outliers with values up to 0.15 . Very young clusters therefore have nearly circular orbits. Then, for clusters older than $30 \mathrm{Myr}$ the dispersion of the eccentricities at a given age is large for both samples. The running median of the HQS shows an increase that is quite smooth at least up to $300 \mathrm{Myr}(\log ($ Age $) \sim 8.5)$. The full sample exhibits a similar behaviour as that of the HQS, but with slightly higher
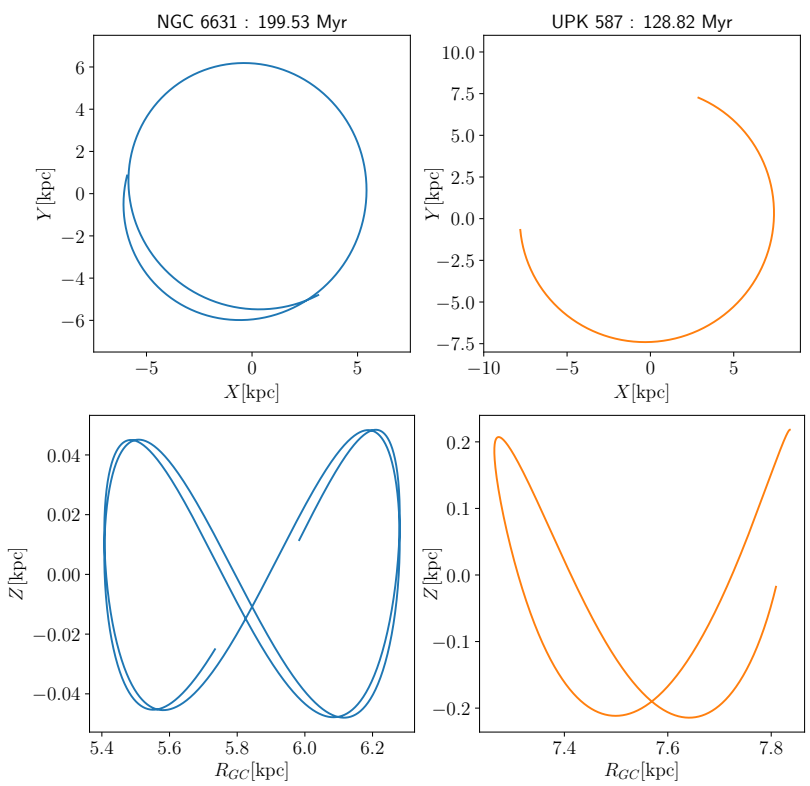

Fig. 12. Example of an orbit for two OCs NGC 6631 and UPK 587 integrated with galpy.

mean eccentricity values. And finnaly, for ages older than $1 \mathrm{Gyr}$ the dispersion in eccentricities starts to be very large for the full sample. For the HQS there seems to be a stabilisation of the eccentricities around a mean value of 0.08 .

This shows that OCs are born on nearly circular orbits, and as their age increases, they are more likely to be gravitationally perturbed from non-axisymmetric components. We did not find very young clusters with high eccentricities, but we found old clusters with both high and low eccentricities. We did not see a preferential location in the Galaxy, or differential characteristics between high- and low-eccentricity old clusters.

\subsection{Action angle variables}

The action-angle variables are a set of canonical coordinates that have been proved to be useful to study the substructure of stars in the $6 \mathrm{D}$ phase space. As extensively discussed by several authors (e.g., McMillan \& Binney 2008), orbital actions $\left(J_{R}, J_{\phi}, J_{z}\right)$ are integrals of motion in an axisymmetric potential, but they also provide information of non-axisymmetric perturbations. In addition, they are independent of time and are therefore more reliable to describe the orbital parameters by removing their time dependence. They have been used to describe stellar components in our Galaxy, in particular, the moving groups seen in the solar neighbourhood in the $(U, V)$ plane (Trick et al. 2019). 

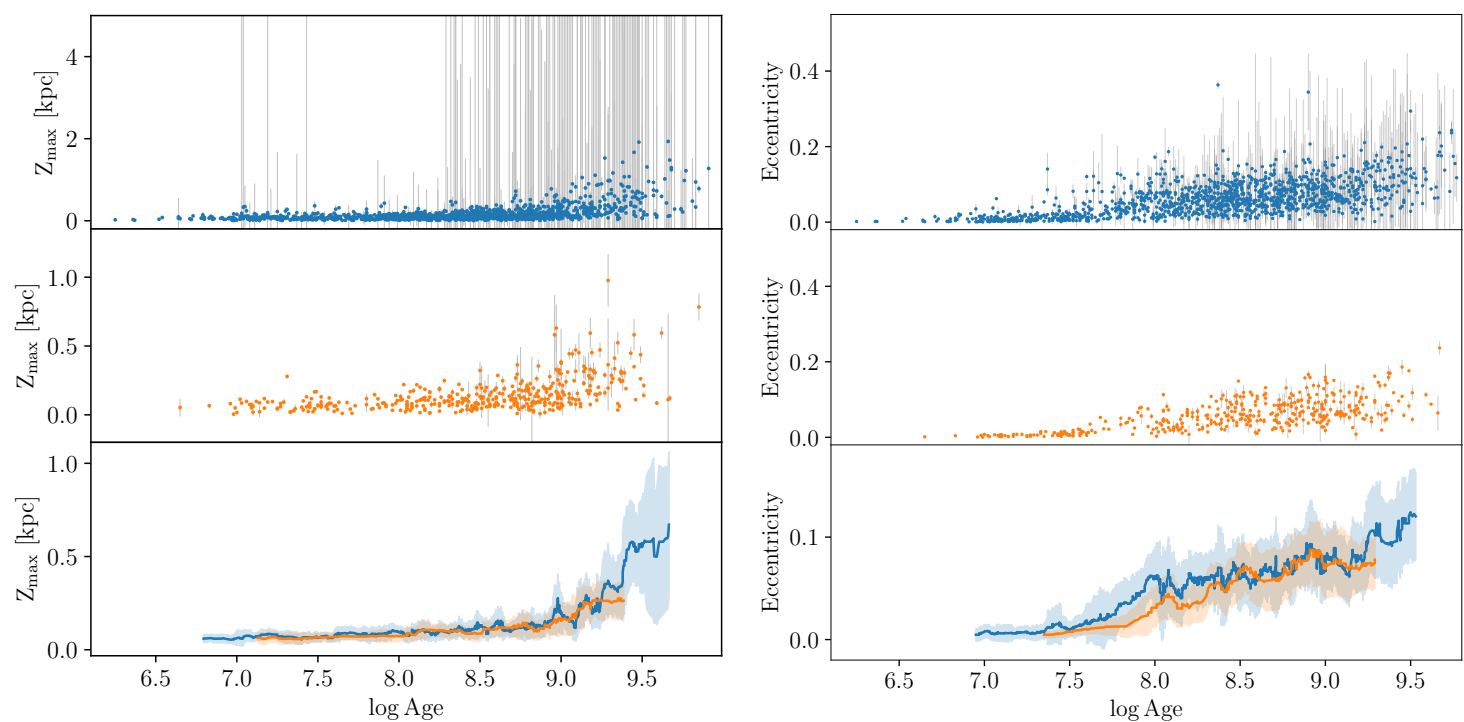

Fig. 13. Maximum altitude above the Galactic plane (left) and eccentricity (right) of the clusters for which we could integrate their orbits as a function of age. In both panels, we represent in blue (in the top panels) the full sample of OCs and in orange (in the middle panels) the HQS. The bottom panels shows the running median of the two samples, calculated with a window of 30 points. The shaded area corresponds to $1 \mathrm{MAD}$ from the median (line). Note the change in scale between the top left panel and the two bottom left panels and between the two top right panels and the bottom right panel.

In an axisymmetric potential, action variables can easily be interpreted as physical quantities. The radial action $J_{R}$ can be used as a proxy for the orbit eccentricity or as a measure of the oscillations around the guiding radius of the object. The azimuthal action $J_{\phi}$ is equal to the angular momentum in the vertical direction $L_{Z}$, which indicates the quantity of rotation of the object around the centre of the Galaxy. Similarly, the last coordinate, the vertical action $J_{z}$, can be used as a measure of the oscillations of the object around the plane of the Galaxy and therefore is a proxy for the maximum height of the object along its orbit.

All of these quantities are conserved in an axisymmetric potential but are affected by non-axisymmetric structures such as a bar or spiral arms, or by a merger. We refer to Sect. 3 of Binney \& Tremaine (2008) for a mathematical and comprehensive description of these variables and their meanings.

We made the computation for the 411 clusters from the HQS with known age. For comparison purposes, we made the same computation with the sample of stars within $d<200 \mathrm{pc}$ in Gaia DR2 RVS, that is, the same selection as Trick et al. (2019), which counts $\sim 350000$ stars.

In Fig. 14 we show the distribution of radial action $J_{R}$ with respect to the vertical component of the angular momentum $L_{Z}$ of field stars compared with the sample of HQS clusters. In all panels, the sample of field stars is the same because we do not know their ages. We indicate the approximate location of the known moving groups present in the solar neighbourhood (Antoja et al. 2008; Gaia Collaboration 2018d), as analysed in the action space by Trick et al. (2019). In this space, the location of a star is interpreted in relation to its orbital characteristics as follows: (i) the $V$ shape is due to the cut in distance $(200 \mathrm{pc})$ made for the sample selection, (ii) stars with circular orbits are placed at $\left(L_{Z}, \sqrt{J_{R}}\right) \sim(1,0)$, while more eccentric orbits appear at larger $J_{R}$, and (iii) stars close to their apocenter (pericenter) are placed in the left (right) edge of the $V$.

We restricted the volume of analysed clusters using their Galactocentric radius instead of their heliocentric distances because of the reduced amount of clusters at $d<200 \mathrm{pc}$. Even though this made the comparison between cluster and field more difficult, the precision in the distances and velocities of the clusters is much better than for individual field stars, so that we expect that the kinematic information is not blurred by this, as usually occurs for individual stars. We made the cuts in $R_{\mathrm{GC}}=8.3 \pm[0.2,0.3,0.5] \mathrm{pc}$, shown in each row. Using the information of cluster ages, we were able to add an additional dimension to the figure, therefore we parted the sample into four age bins $[<30,30-150,150-500,>500]$ Myr, shown as columns. The differences in the volume selection of the clusters and the field stars are visible in the top row, where some of the clusters stand out of the left and right edges, which would correspond to clusters towards the Galactic centre and anticentre.

Figure 14 shows that the bulk of clusters tends to be concentrated towards the position $(1,0)$, which is expected because most OCs have cold kinematics and tend to have nearly circular orbits. All three distance limits (rows) show that there are few clusters at high eccentricities, but they increase in number at older ages. As a consequence, the density peak moves upwards towards older ages. This is particularly clear in the top row because of the large number of points.

Regarding the relation of the clusters and the moving groups, no clusters populate the two Hercules substreams, which are stars with too eccentric orbits and low angular momentum. Conversely, the location of the Hyades, Pleiades, Coma Berenices, and Sirius moving groups more closely resembles the distribution of the clusters, even at larger distances (top row). We highlight that the age bin 30-150 Myr (for all distance cuts) shows a significant clump of clusters towards the middle left side (smaller $L_{z}$ ), populating the region where the Pleiades moving group is found. On the other hand, the Sirius moving group is populated mainly by clusters in the two older age bins. These dependences in the population in each moving group depending on age point in the same direction as the findings by Antoja et al. (2008): in their Fig. 14, the Hyades, Pleiades, and Coma gather most of their young stars $(\$ 300 \mathrm{Myr})$, and at $\gtrsim 300 \mathrm{Myr}$, there is a bump of stars related to Sirius. Hercules is associated 


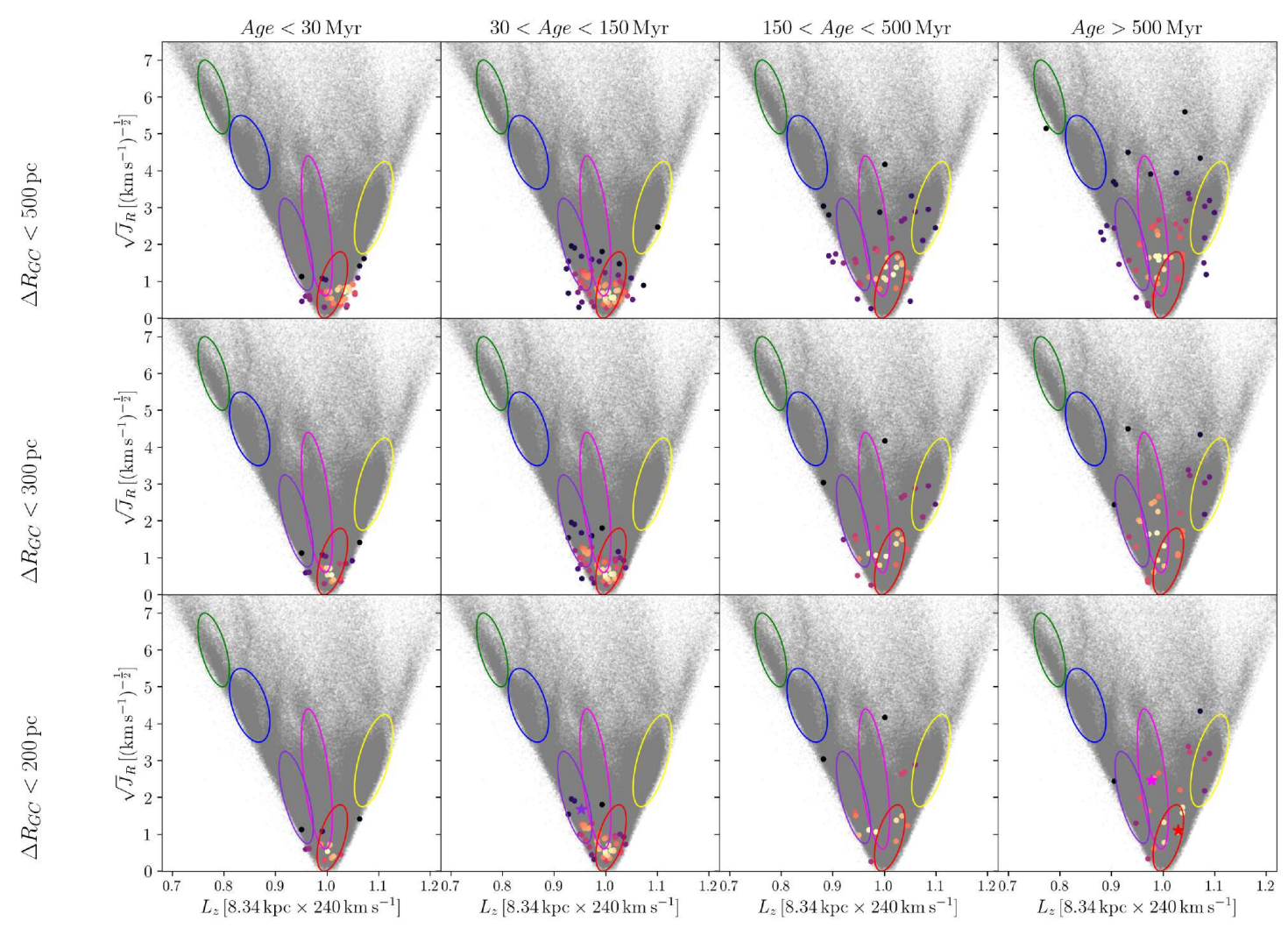

Fig. 14. Radial action vs. angular momentum $\left(J_{R}, L_{z}\right)$ distribution of field stars closer than $200 \mathrm{pc}$ in Gaia DR2/RVS sample (grey), and clusters (coloured dots by the density of points). Each column shows clusters in different age bins, and each row includes clusters selected inside different ranges of Galactocentric radius, instead of heliocentric distances. We indicate the approximate location of the known moving groups analysed by Trick et al. (2019): Hercules (green and blue), Pleiades (dark violet), Hyades (magenta), Coma Berenices (red), and Sirius (yellow). We highlight the values of the three clusters Pleiades, Hyades, and Coma Berenices with a violet (second column in the bottom row), pink, and red star (both in the last column of the bottom row), respectively, in their age- $R_{\mathrm{GC}}$ panels.

with stars of $\sim 2 \mathrm{Gyr}$, and the distributions of all moving groups also exhibit a bump at this age. We find almost no clusters at this old age, however, so there is no indication of this in our sample.

\section{Summary}

By combining Gaia and ground-based surveys and catalogues, and with new memberships from Cantat-Gaudin et al. (2020), we assembled the largest catalogue of RV velocities for OCs in order to study their kinematics. As a by-product of our study, which includes the Gaia DR2 RVS, Gaia-ESO survey, APOGEE, RAVE, GALAH, and smaller catalogues, we compared the RVs from the different sources to each other to assess their typical precision and zero-point. We found RV zero-points to be consistent at a level better than $1 \mathrm{~km} \mathrm{~s}^{-1}$. The scatter of the comparisons indicates that the real precision of each catalogue is compatible with the individual uncertainties listed in it. All non-Gaia RV measurements were corrected to align them on the Gaia RVS zero-point. The weighted mean RV of each star and each cluster resulted in 1382 OCs with an RV, $38 \%$ with a highly reliable RV based on more than three stars and with an uncertainty lower than $3 \mathrm{~km} \mathrm{~s}^{-1}$.

We computed both heliocentric and Galactocentric Cartesian and cylindrical velocities for this sample of OCs and defined a high-quality sample composed of 418 OCs with the most reliable velocities, 411 OCs of which have an age determination. We found that most OCs fall in a band in between the two main arches drawn by field star in the $V_{r}-V_{\phi}$ plane, while they seem to follow the overdensities described by the diagonal ridges in the $R_{\mathrm{GC}}-V_{\phi}$ plane. The rotation curve drawn by our OCs shows two significant dips: at $R_{\mathrm{GC}} \sim 7 \mathrm{kpc}$, and a more prominent one around $R_{\mathrm{GC}} \sim 9.7 \mathrm{kpc}$. The locations and depths of these dips agree with the perturbations we would expect from the non-axisymmetric components of the disc, which also draw the ridges observed in the $R_{\mathrm{GC}}-V_{\phi}$ plane.

With the ages of almost all the clusters from our sample, we investigated the age velocity relation for OCs in detail. It shows a clear anisotropy of the three velocity components. Compared with field stars studies, the heating parameter $\beta$ of OCs was found to be similar in the radial and azimuthal directions, but significantly lower in the vertical direction. This low heating rate in the $Z$ coordinate can be due to the disruption of old clusters, which are the most likely to reach high altitudes above the disc, or to a less efficient heating of OCs by giant molecular clouds. We are aware, however, that the quality cuts we applied discarded distant clusters, resulting in a bias in our sample.

We used the 6D + age information of our sample of OCs to compute and investigate orbits and action variables. We analysed the dependences of the recovered orbital parameters as a function of age. Most of the clusters reach a maximum altitude above the plane during their orbits that is lower than $400 \mathrm{pc}$, and only those older than $1 \mathrm{Gyr}$ are able to move considerably away from the midplane, but typically less than $1 \mathrm{kpc}$. Clusters younger than 30 Myr show a very low eccentricity $(\sim 0.018)$, and for clusters older than this, especially those older than $100 \mathrm{Myr}$, the 
eccentricity shows an increasing relation with age. These results show that OCs are born in circular orbits, and as age increases, they are more prone to suffer perturbations of their orbits. This is also seen after the computation of action variables, where as age increases, the distribution in the $\left(L_{Z}, \sqrt{J_{R}}\right)$ plane tends to spread beyond $\sim(1,0)$. We related our cluster distribution in this action space with the location of the known moving groups as a function of age. We conclude that the Pleiades, Hyades, and Coma moving groups appear to be more populated by young clusters, while the Sirius region appears to have a clump of clusters of age $\gtrsim 300 \mathrm{Myr}$. No clusters populate the two Hercules streams.

Acknowledgements. This work has made use of data from the European Space Agency (ESA) mission Gaia (http://www.cosmos.esa.int/Gaia), processed by the Gaia Data Processing and Analysis Consortium (DPAC, http: // www. cosmos.esa.int/web/Gaia/dpac/consortium). We acknowledge the Gaia Project Scientist Support Team and the Gaia DPAC. Funding for the DPAC has been provided by national institutions, in particular the institutions participating in the Gaia Multilateral Agreement. This research made extensive use of the SIMBAD database, and the VizieR catalogue access tool, operated a the CDS, Strasbourg, France, and of NASA Astrophysics Data System Bibliographic Services. This research has made use of Astropy (Astropy Collaboration 2013), Topcat (Taylor 2005). Y. T., C. S., and L. C. acknowledge support from "programme national de physique stellaire" (PNPS) and from the "programme national cosmologie et galaxies" (PNCG) of CNRS/INSU. L. C. acknowledges the support of the postdoc fellowship from French Centre National d'Etudes Spatiales (CNES). This work has been supported by the Spanish Ministry of Economy (MINECO/FEDER, UE) through grants ESP2016-80079-C2 1-R, RTI2018-095076-B-C21 and the Institute of Cosmos Sciences University of Barcelona (ICCUB, Unidad de Excelencia 'María de Maeztu') through gran MDM-2014-0369 and CEX2019-000918-M. A. B. acknowledges support from Italian MIUR Premiale 2016 "MITiC". A. M. acknowledges the support from the Portuguese FCT Strategic Programme UID/FIS/00099/2019 for CENTRA. This project has received funding from the European Union's Horizon 2020 research and innovation programme under the Marie Skłodowska-Curie grant agreement No. 800502. D. B. acknowledges supported by FCT through the research grants UIDB/04434/2020, UIDP/04434/2020 and PTDC/FIS-AST/30389/2017, and by FEDER - Fundo Europeu de Desenvolvimento Regional through COMPETE2020 -Programa Operacional Competitividade e Internacionalização (grant: POCI-01-0145-FEDER-030389). We warmly thank L. Martínez-Medina for his comments and for sharing with us his models.

\section{References}

Ahumada, R., Allende Prieto, C., Almeida, A., et al. 2020, ApJS, 249, 3 Anguiano, B., Majewski, S. R., Freeman, K. C., Mitschang, A. W., \& Smith, M. C. 2018, MNRAS, 474, 854

Antoja, T., Figueras, F., Fernández, D., \& Torra, J. 2008, A\&A, 490, 135 Antoja, T., Helmi, A., Bienayme, O., et al. 2012, MNRAS, 426, L1

Antoja, T., Helmi, A., Romero-Gómez, M., et al. 2018, Nature, 561, 360 Astropy Collaboration (Robitaille, T. P., et al.) 2013, A\&A, 558, A33 Aumer, M., \& Binney, J. J. 2009, MNRAS, 397, 1286

Aumer, M., Binney, J., \& Schönrich, R. 2016, MNRAS, 462, 1697

Barros, D. A., Lépine, J. R. D., \& Dias, W. S. 2016, A\&A, 593, A108

Barros, D. A., Pérez-Villegas, A., Lépine, J. R. D., Michtchenko, T. A., \& Vieira, R. S. S. 2020, ApJ, 888, 75

Binney, J., \& Tremaine, S. 2008, Galactic Dynamics: Second Edition (Princeton, NJ USA: Princeton University Press)

Bossini, D., Vallenari, A., Bragaglia, A., et al. 2019, A\&A, 623, A108

Boubert, D., Strader, J., Aguado, D., et al. 2019, MNRAS, 486, 2618

Bovy, J. 2015, ApJS, 216, 29

Buder, S., Sharma, S., Kos, J., et al. 2020, MNRAS, submitted [arXiv 2011.02505]

Cantat-Gaudin, T., \& Anders, F. 2020, A\&A, 633, A99

Cantat-Gaudin, T., Jordi, C., Vallenari, A., et al. 2018, A\&A, 618, A93

Cantat-Gaudin, T., Jordi, C., Wright, N. J., et al. 2019, A\&A, 626, A17

Cantat-Gaudin, T., Anders, F., Castro-Ginard, A., et al. 2020, A\&A, 640, A1

Carrera, R., Bragaglia, A., Cantat-Gaudin, T., et al. 2019, A\&A, 623, A80

Casamiquela, L., Carrera, R., Jordi, C., et al. 2016, MNRAS, 458, 3150

Castro-Ginard, A., Jordi, C., Luri, X., et al. 2018, A\&A, 618, A59

Castro-Ginard, A., Jordi, C., Luri, X., Cantat-Gaudin, T., \& Balaguer-Núñez, L. 2019, A\&A, 627, A35
Castro-Ginard, A., Jordi, C., Luri, X., et al. 2020, A\&A, 635, A45

Chereul, E., Crézé, M., \& Bienaymé, O. 1999, A\&AS, 135, 5

Cui, X.-Q., Zhao, Y.-H., Chu, Y.-Q., et al. 2012, Res. Astron. Astrophys., 12, 1197

Dehnen, W., \& Binney, J. J. 1998, MNRAS, 298, 387

Dias, W. S., Alessi, B. S., Moitinho, A., \& Lépine, J. R. D. 2002, A\&A, 389, 871 Dias, W. S., Monteiro, H., Lépine, J. R. D., \& Barros, D. A. 2019, MNRAS, 486, 5726

Eggen, O. J. 1958, MNRAS, 118, 65

Eggen, O. J. 1996, AJ, 112, 1595

Famaey, B., Siebert, A., \& Jorissen, A. 2008, A\&A, 483, 453

Ferreira, F. A., Santos, J. F. C., Corradi, W. J. B., Maia, F. F. S., \& Angelo, M. S. 2019, MNRAS, 483, 5508

Freeman, K. C. 1987, ARA\&A, 25, 603

Gaia Collaboration (Brown, A. G. A., et al.) 2018a, A\&A, 616, A1

Gaia Collaboration (Katz, D., et al.) 2018b, A\&A, 616, A11

Gaia Collaboration (Babusiaux, C., et al.) 2018c, A\&A, 616, A10

Gaia Collaboration (Helmi, A., et al.) 2018d, A\&A, 616, A12

Grand, R. J. J., Springel, V., Gómez, F. A., et al. 2016, MNRAS, 459, 199

Gustafsson, B., Church, R. P., Davies, M. B., \& Rickman, H. 2016, A\&A, 593, A85

Jackson, R. J., Jeffries, R. D., Lewis, J., et al. 2015, A\&A, 580, A75

Jenkins, A. 1992, MNRAS, 257, 620

Jenkins, A., \& Binney, J. 1990, MNRAS, 245, 305

Katz, D., Sartoretti, P., Cropper, M., et al. 2019, A\&A, 622, A205

Kawata, D., Baba, J., Ciucă, I., et al. 2018, MNRAS, 479, L108

Khanna, S., Sharma, S., Tepper-Garcia, T., et al. 2019, MNRAS, 489, 4962

Kharchenko, N. V., Piskunov, A. E., Schilbach, E., Röser, S., \& Scholz, R. D. 2013, A\&A, 558, A53

Krone-Martins, A., \& Moitinho, A. 2014, A\&A, 561, A57

Kuijken, K., \& Tremaine, S. 1994, ApJ, 421, 178

Lacey, C. G. 1984, MNRAS, 208, 687

Liu, L., \& Pang, X. 2019, ApJS, 245, 32

Mackereth, J. T., Bovy, J., Leung, H. W., et al. 2019, MNRAS, 489, 176

Martig, M., Minchev, I., \& Flynn, C. 2014, MNRAS, 443, 2452

Martinez-Medina, L., Pichardo, B., Peimbert, A., \& Valenzuela, O. 2019, MNRAS, 485, L104

McGaugh, S. S. 2019, ApJ, 885, 87

McMillan, P. J. 2017, MNRAS, 465, 76

McMillan, P. J., \& Binney, J. J. 2008, MNRAS, 390, 429

Mermilliod, J. C., Mayor, M., \& Udry, S. 2008, A\&A, 485, 303

Mermilliod, J.-C., Mayor, M., \& Udry, S. 2009, A\&A, 498, 949

Miyamoto, M., \& Nagai, R. 1975, PASJ, 27, 533

Monari, G., Famaey, B., Minchev, I., et al. 2018, Res. Notes Am. Astron. Soc., 2,32

Mróz, P., Udalski, A., Skowron, D. M., et al. 2019, ApJ, 870, L10

Nakanishi, H., \& Sofue, Y. 2016, PASJ, 68, 5

Navarro, J. F., Frenk, C. S., \& White, S. D. M. 1997, ApJ, 490, 493

Nordström, B., Mayor, M., Andersen, J., et al. 2004, A\&A, 418, 989

Piatti, A. E. 2020, A\&A, 639, A55

Randich, S., \& Gilmore, G., \& Gaia-ESO Consortium 2013, Messenger, 154, 47

Reid, M. J., Menten, K. M., Brunthaler, A., et al. 2014, ApJ, 783, 130

Reid, M. J., Menten, K. M., Brunthaler, A., et al. 2019, ApJ, 885, 131

Sartoretti, P., Katz, D., Cropper, M., et al. 2018, A\&A, 616, A6

Schönrich, R., Binney, J., \& Dehnen, W. 2010, MNRAS, 403, 1829

Sharma, S., Hayden, M. R., Bland-Hawthorn, J., et al. 2020, MNRAS, submitted [arXiv: 2004.06556]

Sim, G., Lee, S. H., Ann, H. B., \& Kim, S. 2019, J. Korean Astron. Soc., 52, 145

Sofue, Y. 2020, Galaxies Rev., 8, 37

Sofue, Y., Honma, M., \& Omodaka, T. 2009, PASJ, 61, 227

Soubiran, C., Bienaymé, O., Mishenina, T. V., \& Kovtyukh, V. V. 2008, A\&A, 480, 91

Soubiran, C., Jasniewicz, G., Chemin, L., et al. 2013, A\&A, 552, A64

Soubiran, C., Cantat-Gaudin, T., Romero-Gómez, M., et al. 2018a, A\&A, 619, A155

Soubiran, C., Jasniewicz, G., Chemin, L., et al. 2018b, A\&A, 616, A7

Spitzer, L. J., \& Schwarzschild, M. 1951, ApJ, 114, 385

Steinmetz, M., Zwitter, T., Matijevic, G., Siviero, A., \& Munari, U. 2018, Res. Notes Am. Astron. Soc., 2, 194

Steinmetz, M., Guiglion, G., McMillan, P. J., et al. 2020, AJ, 160, 83

Taylor, M. B. 2005, ASP Conf. Ser., 347, 29

Trick, W. H., Coronado, J., \& Rix, H.-W. 2019, MNRAS, 484, 3291

Wielen, R. 1977, A\&A, 60, 263

Worley, C. C., de Laverny, P., Recio-Blanco, A., et al. 2012, A\&A, 542, A48

Xiang, M. S., Liu, X. W., Yuan, H. B., et al. 2015, MNRAS, 448, 822

Yu, J., \& Liu, C. 2018, MNRAS, 475, 1093 